\title{
ON THE DISTRIBUTION OF STELLAR REMNANTS AROUND MASSIVE BLACK HOLES: SLOW MASS SEGREGATION, STAR CLUSTER INSPIRALS, AND CORRELATED ORBITS
}

\author{
FABio Antonini \\ Canadian Institute for Theoretical Astrophysics, University of Toronto, 60 St. George Street, Toronto, Ontario M5S 3H8, Canada; antonini@ cita.utoronto.ca \\ Received 2014 February 19; accepted 2014 August 13; published 2014 September 29
}

\begin{abstract}
We use $N$-body simulations as well as analytical techniques to study the long-term dynamical evolution of stellar black holes (BHs) at the Galactic center (GC) and to put constraints on their number and mass distribution. Starting from models that have not yet achieved a state of collisional equilibrium, we find that timescales associated with cusp regrowth can be longer than the Hubble time. Our results cast doubts on standard models that postulate high densities of BHs near the GC and motivate studies that start from initial conditions that correspond to well-defined physical models. For the first time, we consider the distribution of BHs in a dissipationless model for the formation of the Milky Way nuclear cluster (NC), in which massive stellar clusters merge to form a compact nucleus. We simulate the consecutive merger of $\sim 10$ clusters containing an inner dense sub-cluster of BHs. After the formed NC is evolved for $\sim 5 \mathrm{Gyr}$, the BHs do form a steep central cusp, while the stellar distribution maintains properties that resemble those of the GC NC. Finally, we investigate the effect of BH perturbations on the motion of the GC S-stars as a means of constraining the number of the perturbers. We find that reproducing the quasi-thermal character of the S-star orbital eccentricities requires $\gtrsim 1000 \mathrm{BHs}$ within $0.1 \mathrm{pc}$ of Sgr A*. A dissipationless formation scenario for the GC NC is consistent with this lower limit and therefore could reconcile the need for high central densities of BHs (to explain the S-stars orbits) with the "missing-cusp" problem of the GC giant star population.
\end{abstract}

Key words: galaxies: nuclei - Galaxy: center - Galaxy: formation - stars: black holes stars: kinematics and dynamics

Online-only material: color figures

\section{INTRODUCTION}

Over the whole Hubble sequence, massive nuclear clusters (NCs) are observed at the center of many galaxies. The frequency of nucleation among galaxies less luminous than $\sim 10^{10.5} L_{\odot}$ is close to $90 \%$ as determined by ACS HST observations of galaxies in the Virgo and Fornax galaxy clusters (Carollo et al. 1998; Böker et al. 2002; Côté et al. 2006; Turner et al. 2012). The study of NCs is of great interest for our understanding of galaxy formation and evolution as indicated by the fact that a number of fairly tight correlations are observed between their masses and global properties of their host galaxies such as velocity dispersion and bulge mass (Ferrarese et al. 2006; Wehner \& Harris 2006; Graham \& Spitler 2009; Scott \& Graham 2013; Leigh et al. 2012). Intriguingly, similar scaling relations are obeyed by massive black holes (MBHs), which are predominantly found in massive galaxies that, however, show little evidence of nucleation (e.g., Graham \& Spitler 2009; Neumayer \& Walcher 2012). The existence of such correlations might indicate a direct link among large galactic spatial scales and the much smaller scale of the nuclear environment and suggests that NCs contain information about the processes that have shaped the central regions of their host galaxies.

How NC formation takes place at the center of galaxies is still largely debated (e.g., Hartmann et al. 2011; Gnedin et al. 2014; Carlberg \& Hartwick 2014; Mastrobuono-Battisti et al. 2014). Relatively recent work has shown that "dissipationless" models can reproduce without obvious difficulties the observed properties (Turner et al. 2012) and scaling relations (Antonini 2013) of NCs. In these models an NC forms through the inspiral of massive stellar clusters into the center because of dynamical friction where they merge to form a compact nucleus (e.g., Tremaine et al. 1975; Capuzzo-Dolcetta \& Miocchi 2008;
Capuzzo-Dolcetta 1993). Alternatively, NCs could have formed locally as a result of radial gas inflow into the galactic center accompanied by efficient dissipative processes (Schinnerer et al. 2008; Milosavljević 2004). Naturally, dissipative and dissipationless processes are not exclusive and both could be important for the formation and evolution of NCs (Hartmann et al. 2011; Antonini et al. 2012; De Lorenzi et al. 2013).

The Milky Way NC, being only $8 \mathrm{kpc}$ away, is currently the only NC that can be resolved in individual stars and for which a kinematical structure and density profile can be reliably determined (Genzel et al. 2010). This offers the unique possibility to resolve the stellar population in order to study the composition and dynamics close to an $\mathrm{MBH}$ and put constraints on different NC formation scenarios. The Milky Way NC has an estimated mass of $\sim 10^{7} M_{\odot}$ (Launhardt et al. 2002; Schödel et al. 2009), and it hosts a massive black hole of $\sim 4 \times 10^{6} M_{\odot}$ (Genzel et al. 2003; Ghez et al. 2008; Gillessen 2009), whose gravitational potential dominates over the stellar cusp potential out to a radius of roughly $3 \mathrm{pc}$ - the $\mathrm{MBH}$ radius of influence. A handful of other galaxies are also known to contain both an NC and an MBH, which typically have comparable masses (Seth et al. 2008). Population synthesis models suggest that roughly $80 \%$ of the stellar mass in the inner parsec of the Milky Way is in (>5 Gyr) old stars (Pfuhl et al. 2011) although the light is dominated by the young stars. This appears to also typically be the case in most NCs observed in external galaxies (Rossa et al. 2006).

Over the last decade, observations of the Galactic NC have led to a number of puzzling discoveries. These discoveries include the presence of a young population of stars (the $\mathrm{S}$ stars) near Sgr A* in an environment extremely hostile to star formation (paradox of youth; Morris 1993; Schödel et al. 2002) and a significant paucity of red giant stars in the inner half of a 
parsec (conundrum of old age; Merritt 2010). Number counts of the giant stars at the Galactic center (GC) show that their visible distribution is in fact quite inconsistent with the distribution of stars expected for a dynamically relaxed population near a dominating Keplerian potential (Buchholz et al. 2009; Do et al. 2009; Bartko et al. 2010): instead of a steeply rising Bahcall $\&$ Wolf (1976) cusp, there is a $\sim 0.5$ pc core. The lack of a Bahcall-Wolf cusp in the giant distribution casts doubts on dynamically relaxed, quasi-steady-state models of the GC that postulate a high central density of stars and stellar black holes (BHs). In these models the central distribution of stars and BHs is determined by just a handful of parameters: the MBH mass, the total density outside the relaxed region, and the slope of the initial mass function (IMF; Merritt 2013). Given the unrelaxed form of the density profile of stars, making predictions about the distribution of the stellar remnants becomes a much more challenging, time-dependent problem susceptible to the initial conditions and to the (yet largely unconstrained) formation process of the NC (Antonini \& Merritt 2012).

Understanding the distribution of the "stellar remnants" in systems similar to the Milky Way's NC is crucial in many respects. Examples include randomization of the S-star orbits via gravitational encounters (Perets et al. 2009), warping of the young stellar disk (Kocsis \& Tremaine 2011), and formation of X-ray binaries (Muno et al. 2005). Stellar nuclei similar to that of the Milky Way are also the location of astrophysical processes that are potential gravitational wave $(\mathrm{GW})$ sources both for ground- and space-based laser interferometers. These include the merger of compact object binaries near MBHs (Antonini \& Perets 2012) and the capture of BHs by MBHs, called "extreme mass ratio inspirals" (EMRIs; Amaro-Seoane et al. 2012). The efficiency of these dynamical processes and rate estimates for GW sources are very sensitive to the number of BHs near the center. Therefore, a fundamental question is whether, when given a prediction for the initial distribution of stars and BHs, the system is old enough that the heavy remnants had time to relax and segregate to the center of the Galaxy.

Motivated by the above arguments, we consider the long-term evolution of $\mathrm{BH}$ populations at the center of galaxies, starting from different assumptions regarding their initial distribution. Since the stellar BHs at the GC are not directly detected, timedependent numerical calculations, like the ones presented below, are crucial for understanding and making predictions about the distribution of stellar remnants at the center of galaxies.

In Section 2 we explore the evolution of models in which stars and BHs follow initially the same spatial distribution, which is far from being in collisional equilibrium. Contrary to some previous claims (Preto \& Amaro-Seoane 2010), we find that in these models the time to regrow a cusp in both the $\mathrm{BH}$ and the star distribution is longer than the age of the Galaxy. For realistic number fractions of $\mathrm{BHs}$, our simulations demonstrate that over the age of the Galaxy, the presence of a heavy component has little effect on the evolution of the stellar component.

In Sections 3, 4, and 5, we discuss the evolution of BHs in a globular cluster merger model for NCs. We present the results of direct $N$-body simulations of the merger of globular clusters containing two mass populations: stars and BHs. These systems were in an initial state of mass segregation with the $\mathrm{BH}$ population concentrated toward the cluster core. Each cluster was placed on a circular orbit with a galactocentric radius of $20 \mathrm{pc}$ in a $N$-body system containing a central $\mathrm{MBH}$. We find that the inspiral of massive globular clusters in the center of the Galaxy constitutes an efficient source term of BHs in these regions. After about 10 inspiral events, the BHs are highly segregated to the center. After a small fraction of the nucleus relaxation time (as defined by the main stellar population), the BHs attain a nearly steady-state distribution; at the same time the stellar density profile exhibits a $\sim 0.2 \mathrm{pc}$ core, similar to the size of the core in the distribution of stars at the GC. Our results indicate that standard models, which assume the same initial phase space distribution for BHs and stars, can lead to misleading results regarding the current dynamical state of the Galactic center.

We discuss the implications of our results in Section 6. In particular, we show that in order to reproduce the quasi-thermal form of the observed eccentricity distribution of the S-star orbits, about 1000 BHs should be present inside $\sim 0.1$ pc of Sgr A*. This number appears to be consistent with the number of $\mathrm{BHs}$ expected in a model in which the Milky Way NC formed through the orbital decay and merger of about 10 massive clusters.

Our main results are summarized in Section 7.

\section{SLOW MASS SEGREGATION AT THE GALACTIC CENTER}

In this section we study the long-term dynamical evolution of multi-mass models for the Milky Way NC. The primary goal of this study is to understand the evolution of the distribution of stars and BHs over a time of order the central relaxation time of the nucleus, starting from initial conditions that are far from being in collisional equilibrium.

\subsection{Evolution toward the Steady State}

We consider four mass groups representing main-sequence stars (MSs), white dwarfs (WDs), neutron stars (NSs), and BHs. After the quasi steady state is attained, the stars are expected to follow a central $r^{-3 / 2}$ cusp, while the heavier particles will have a steeper $r^{-2}$ density profile (e.g., Alexander 2005). We assume that all species have the same phase space distribution initially as would be expected for a violently relaxed system. This is the assumption that was made in most previous papers (e.g., Freitag et al. 2006; Hopman \& Alexander 2006; Merritt 2010). We specify the mass ratio, $m_{\mathrm{wd}} / m_{\star}=0.6, m_{\mathrm{ns}} / m_{\star}=1.4$, and $m_{\mathrm{bh}} / m_{\star}=10$, between the mass group particles and respective number fractions, $f_{\mathrm{wd}}=N_{\mathrm{wd}} / N_{\star}, f_{\mathrm{ns}}=N_{\mathrm{ns}} / N_{\star}$, and $f_{\text {bh }}=N_{\text {bh }} / N_{\star}$.

Number counts of the old stellar population at the GC are consistent with a density profile of stars that is flat or slowly rising toward the $\mathrm{MBH}$ inside its sphere of influence and within a radius of roughly $\sim 0.5$ pc (Buchholz et al. 2009; Do et al. 2009; Bartko et al. 2010). Outside this radius the density falls off as $r^{-2}$. Merritt (2010) showed that a core of size $\sim 0.5 \mathrm{pc}$ is a natural consequence of two-body relaxation acting over $10 \mathrm{Gyr}$, starting from a core of radius $\sim 1 \mathrm{pc}$. It is therefore of interest to study the evolution of the $\mathrm{BH}$ distribution for a time of order the age of the Galaxy and starting from a density distribution with a parsecscale core. We adopt the truncated broken-power-law model:

$$
\rho(r)=\rho_{0}\left(\frac{r}{r_{0}}\right)^{-\gamma_{i}}\left[1+\left(\frac{r}{r_{0}}\right)^{\alpha}\right]^{\left(\gamma_{i}-\gamma_{e}\right) / \alpha} \zeta\left(r / r_{\text {cut }}\right),
$$

where $\zeta(x)=(2 / \operatorname{sech}(x)+\cosh (x)), \alpha$ is a parameter that defines the transition strength between inner and outer power laws, $r_{0}$ is the scale radius, and $r_{\text {cut }}$ is the truncation radius of the model. The values adopted for these parameters were 
$r_{0}=1.5 \mathrm{pc}, \alpha=4, \gamma_{e}=1.8$, and $r_{\text {cut }}=6$ pc. We included a central MBH of mass $M_{\bullet}=4 \times 10^{6} M_{\odot}$ and generated the models $N$-body representations via numerically calculated distribution functions. The central slope was set to $\gamma_{i}=0.6$, the smallest density slope index consistent with an isotropic distribution for the adopted density model and potential.

The normalizing factor $\rho_{0}$ was chosen in such a way that the corresponding density profile reproduces the coreless density model

$$
\rho(r)=1.5 \times 10^{5}\left(\frac{r}{1 \mathrm{pc}}\right)^{-1.8} M_{\odot} \mathrm{pc}^{-3}
$$

outside the core. This choice of normalizing constant gives a mass density at $1 \mathrm{pc}$ similar to what is inferred from observations (e.g., Oh et al. 2009) and gives a total mass in stars within this radius of $\sim 1.6 \times 10^{6} M_{\odot}$. The fact that our models are directly scalable to the observed stellar density distribution of stars at the GC is important if we want to draw conclusions about the current dynamical state of stars and BHs at the GC. We note, for example, that the merger models of Gualandris \& Merritt (2012) had core radii that were substantially larger than the $\mathrm{MBH}$ influence radius. As also noted by these authors, this simple fact precluded a unique scaling of their models to the Milky Way - at least in the Galaxy's current state in which the stellar core size $(\sim 0.5 \mathrm{pc})$ is much smaller than the Sgr A* influence radius $(\sim 3 \mathrm{pc})$.

We run three simulations with $N=132 \mathrm{k}$ particles. These simulations differ with each other by the adopted number fractions of the four mass groups: (1) $f_{\mathrm{wd}}=f_{\mathrm{ns}}=f_{\mathrm{bh}}=0$; (2) $f_{\mathrm{wd}}=10^{-1}, f_{\mathrm{ns}}=10^{-2}, f_{\mathrm{bh}}=10^{-3}$; and (3) $f_{\mathrm{wd}}=2 \times 10^{-1}$, $f_{\mathrm{ns}}=2 \times 10^{-2}, f_{\mathrm{bh}}=5 \times 10^{-3}$. The latter two set of values correspond roughly to the number fractions expected from a standard and from a top-heavy IMF, respectively. A fraction of $f_{\text {bh }}=10^{-3}$ is what is expected for a standard (Kroupa-like) IMF, and it is the value typically adopted in previous studies (e.g., Hopman \& Alexander 2005, 2006). Although a larger fraction of stellar remnants might be possible, for instance, if the Galactic center always obeyed a top-heavy initial mass function, the observationally constrained mass-to-light ratio of the inner parsec limits the BH fraction to only a few percent and is more consistent with a ratio and a total mass of $\mathrm{BHs}$ predicted by a standard IMF (Löckmann et al. 2010). We evolved these systems for a time equal to the relaxation time, $T_{r_{\text {inf }}}$, computed at the sphere of influence of the $\mathrm{MBH}$. The relaxation time was evaluated using the expression (Spitzer 1987):

$$
T_{r}=\frac{0.34 \sigma(r)^{3}}{G^{2}\langle m\rangle \ln \Lambda \rho(r)},
$$

where $\rho$ is the total local mass density, and $\langle m\rangle$ is the average particle mass. For the Coulomb logarithm we used $\ln \Lambda=$ $\ln \left(r_{\text {infl }} \sigma^{2} / 2 G m_{\star}\right) \approx 10$, with $\sigma$ the $1 \mathrm{~d}$ velocity dispersion outside $r_{\text {infl }}=G M_{\bullet} / \sigma^{2}$.

To scale the $N$-body time length to the Milky Way, we consider that the relaxation time at the influence radius of $\operatorname{Sgr} \mathrm{A}^{*}$, $r_{\text {infl }} \approx 3 \mathrm{pc}$, is $T_{r_{\text {inf }}} \approx 25 \mathrm{Gyr}$, assuming a stellar mass of $1 M_{\odot}$ (Merritt 2010; Antonini \& Merritt 2012). Thus, when scaling to the GC, a time of $0.4 T_{r_{\text {inf }}}$ corresponds to roughly $10 \mathrm{Gyr}$.

We evolved the initial conditions with the direct $N$-body integrator $\phi$ GRAPEch (Harfst et al. 2008). The code implements a fourth-order Hermite integrator with a predictorcorrector scheme and hierarchical time-stepping. The code combines hardware-accelerated computation of pairwise interparticle forces (using the Sapporo library, which emulates the
GRAPE interface utilizing GPU boards; Gaburov et al. 2009) with a high-accuracy chain regularization algorithm to follow the dynamical interactions of field particles with the central $\mathrm{MBH}$ particle. The chain radius was set to $10^{-2} \mathrm{pc}$, and we used a softening $\epsilon=10^{-6} \mathrm{pc}$. The relative error in total energy was typically $\sim 10^{-4}$ for the accuracy parameter $\eta=0.01$.

Figure 1 shows the evolution of the $N$-body models over one relaxation time. The heavy particles segregate to the center because of dynamical friction. After the central mass density of $\mathrm{BHs}$ becomes comparable to the density in the other species, the evolution of the $\mathrm{BH}$ population starts being dominated by $\mathrm{BH}-\mathrm{BH}$ self-interactions; at the same time the lighter species evolve in response to dynamical heating from the BHs, which causes the local stellar densities to decrease and Lagrangian radii to expand. As shown below, the same heating rapidly converts the initial density profile into a steeply rising density cusp with slope, $\gamma \equiv-d \log \rho / d \log r \approx 3 / 2$. The inclusion of a BH population has therefore two effects on the main-sequence population: it lowers the stellar densities and at the same time it accelerates the evolution of the density of stars toward the $\gamma=3 / 2$ steady-state form.

The lower panels of Figure 1 display the density profile of stars and BHs over 10 Gyr of evolution. These plots show that, starting with a fraction of $\mathrm{BHs}$ that corresponds to a standard IMF, (1) after $\sim 10$ Gyr the density of BHs can remain well below the density of stars at all radii, and (2) even after $10 \mathrm{Gyr}$ of evolution, the density distribution of stars looks very different from what expected for a dynamically relaxed population around an $\mathrm{MBH}$. These findings are in agreement with the Fokker-Plank simulations of Merritt (2010) but in contrast with more recent claims that mass segregation can rebuild a stellar cusp in a relatively small fraction of the Hubble time (e.g., Preto \& Amaro-Seoane 2010, and the Introduction of Chen \& Amaro-Seoane 2014). Figure 2 displays the evolution of the radial profile of the density profile slope. Comparing the evolution observed in models with and without BHs, we see that a cusp in the main-sequence population develops earlier in models with BHs. Figure 2 shows that for $f_{\mathrm{bh}}=10^{-3}$ and $f_{\mathrm{bh}}=5 \times 10^{-3}$, a stellar cusp only develops after $\sim 0.6 T_{r_{\text {inf }}}$ and $\sim 0.4 T_{r_{\text {inf }}}$, respectively. Therefore, over the timescales $(\lesssim 10 \mathrm{Gyr})$ and radii $(r \gtrsim 0.01 \mathrm{pc})$ of relevance, the inclusion of a BH population has little or even no influence on the evolution of the lighter populations. This latter point is more clearly demonstrated in Figure 3, which directly compares the Lagrangian radii evolution of our $f_{\text {bh }}=0$ model with models with BHs. The stellar populations evolve similarly in these models independently of $f_{\text {bh }}$ until approximately 0.6 and $0.4 \times T_{r_{\text {inf }}}$ for $f_{\mathrm{bh}}=10^{-3}$ and $5 \times 10^{-3}$, respectively. After this time, heating of the lighter species by the heavy particles starts becoming important, causing the density of the former to decrease and deviate from the evolution observed in the single-mass component model. However, the transition to this phase clearly occurs after the models have been already evolved for a time comparable to (for $f_{\mathrm{bh}}=5 \times 10^{-3}$ ) or longer than (for $f_{\mathrm{bh}}=10^{-3}$ ) the age of the Galactic NC. ${ }^{1}$

Given the results of the simulations presented in this section, we can schematically divide mass segregation in two phases: in phase 1 the density of BHs is smaller than the density of stars and the models evolve mainly because of scattering off the stars - the $\mathrm{BHs}$ inspiral to the center because of dynamical friction and the stellar distribution relaxes as in a single-mass component model.

\footnotetext{
1 The mean stellar age in the Galactic NC is estimated to be $\sim 5$ Gyr (Figer
} et al. 2004). 


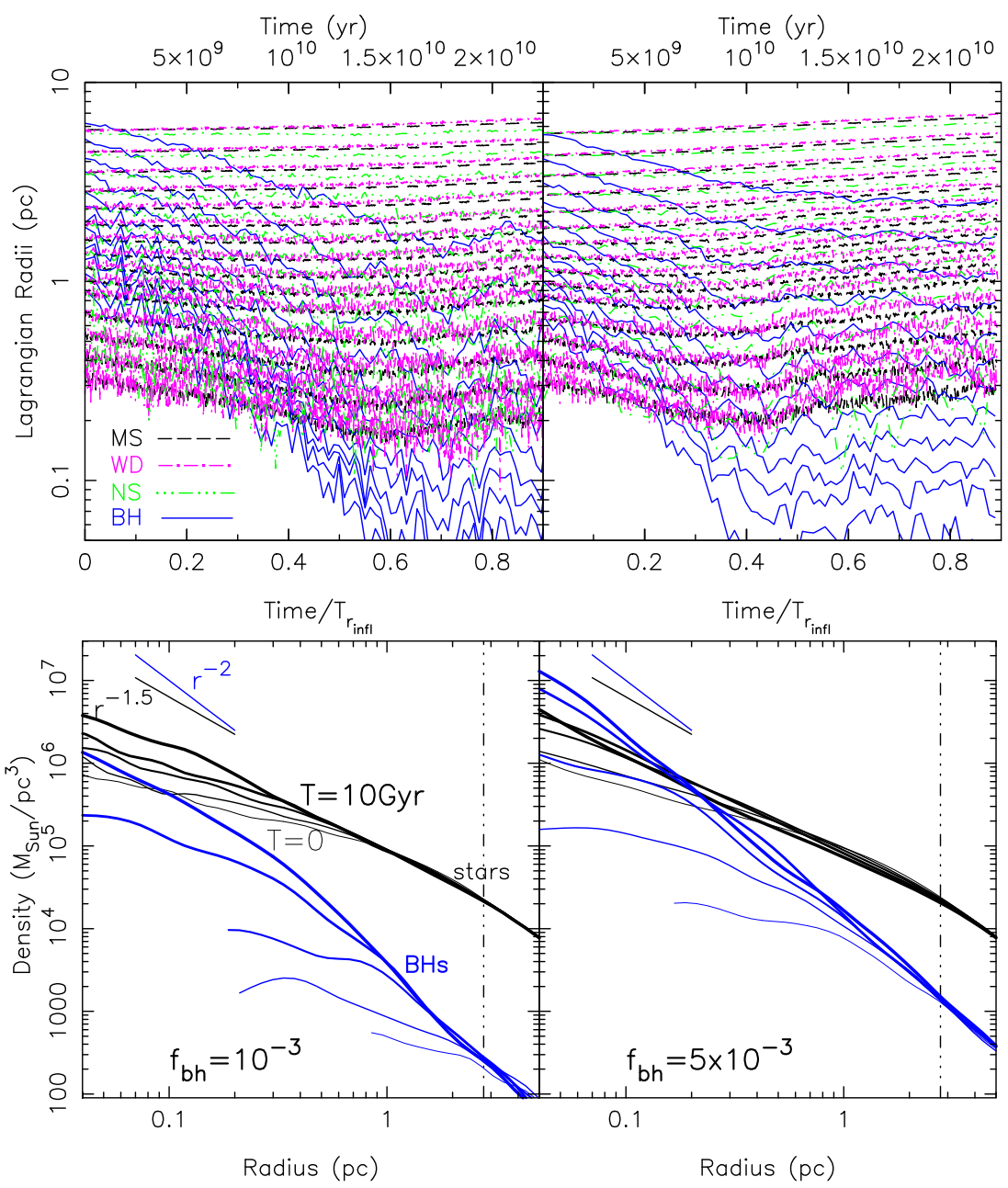

Figure 1. Top panels show the Lagrangian radii for the four stellar species during the $N$-body simulations with BH number fractions: $f_{\mathrm{bh}}=10^{-3}$ (left panels) and $f_{\text {bh }}=5 \times 10^{-3}$ (right panels). Top tick marks give times after scaling to the Milky Way; we adopted a relaxation time at the Sgr A* influence radius of 25 Gyr (e.g., Merritt 2010). Bottom panels show the density profile of stars and BHs at $t=(0,2.5,5,7.5,10) \mathrm{Gyr}$; the central density increases with time. Clearly, even after a time of the order of $10 \mathrm{Gyr}$, the distribution of stars and BHs in our models can be very different from the relaxed multi-mass models that are often used to describe the center of galaxies. The vertical line marks the MBH influence radius.

(A color version of this figure is available in the online journal.)


Figure 2. Evolution of the density slope, $\gamma \equiv-d \log \rho / d \log r$, of the main-sequence density profile in multi-mass $N$-body models (center and right panels) compared with a model with only one mass component (left panel). The continued curves show profiles at $(0.2,0.4,0.6,0.8,1) \times T_{r_{\text {inf }}}$; increasing line width corresponds to increasing time. The dashed curve corresponds to the initial model. Adding a BH component accelerates the growth of a density cusp in the stellar component. However, the time to regrow a cusp in these models is always longer than $0.2 T_{r_{\text {infl }}}$, i.e., 5 Gyr when scaled to the Milky Way, a time longer than the mean stellar age of the Galactic NC. 

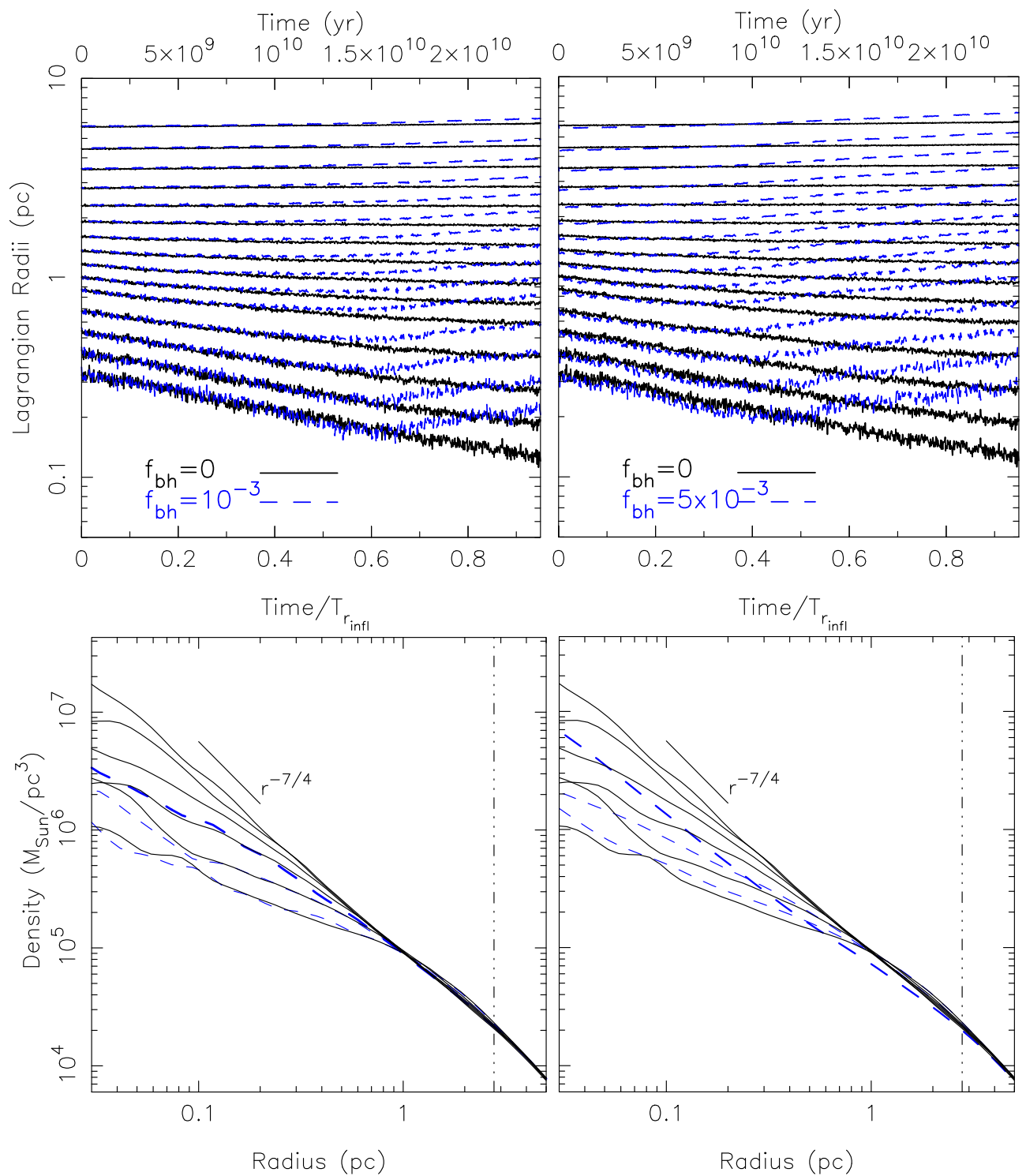

Figure 3. Evolution of the $f_{\mathrm{bh}}=0$ model Lagrangian radii and density profile compared with the evolution of models with $f_{\mathrm{bh}}=10^{-3}\left(\right.$ left panel) and $f_{\mathrm{bh}}=5 \times 10^{-3}$ (right panel). In the bottom panels the density profile is plotted at $t=(0,5,10,15,20,25) \mathrm{Gyr}$ of evolution. The blue dashed curves give the evolution of the stellar distribution Lagrangian radii in the models with BHs, and the bottom panels show the respective stellar density profiles at $t=(0,5,10)$ Gyr. Over a time of order $10 \mathrm{Gyr}$, the evolution of the density profile of stars in the $f_{\mathrm{bh}}=10^{-3}$ model is not much affected by the presence of the BHs. In the model with $f_{\mathrm{bh}}=5 \times 10^{-3}$ the evolution toward the steady state is faster, and after $10 \mathrm{Gyr}$ the stars have formed a cusp. Vertical lines give the MBH influence radius.

(A color version of this figure is available in the online journal.)

In phase 2 , when the density of BHs becomes comparable and larger than the density of stars, BHs and stars evolve because of scattering off the BHs, which causes the models to rapidly evolve toward the steady state.

Perhaps the most interesting aspect of our simulations is the long timescale required by the $\mathrm{BH}$ population to segregate to the center through dynamical friction (phase 1 above) and reach a (nearly) steady-state distribution - a time comparable to the relaxation time as defined by the dominant stellar population. In what follows we show that these predictions agree well with the evolution expected on the basis of theoretical arguments.

\subsection{Analytical Estimates}

In order to understand the evolution of the distribution of BHs observed in the $\mathrm{N}$-body simulations, we evolved the population of massive remnants using an analytical estimate for the dynamical friction coefficient. The stellar background was represented as an analytic potential that was also allowed to evolve in time according to the evolution observed in the stellar distribution during the $N$-body simulations.

We began by generating random samples of positions and velocities from the isotropic distribution function corresponding to the density model of Equation (1). The orbital equations of motion were then integrated forward in time in the evolving smooth stellar potential and included a term that describes the orbital energy dissipation due to dynamical friction. The dynamical friction acceleration was computed using the expression

$$
\begin{aligned}
\boldsymbol{a}_{\mathrm{fr}} \approx & -4 \pi G^{2} m_{\mathrm{bh}} \rho(r) \frac{\boldsymbol{v}}{v^{3}}\left(\ln \Lambda \int_{0}^{v} d v_{\star} 4 \pi f\left(v_{\star}\right) v_{\star}^{2}\right. \\
& \left.+\int_{v}^{\infty} d v_{\star} 4 \pi f\left(v_{\star}\right) v_{\star}^{2}\left[\ln \left(\frac{v_{\star}+v}{v_{\star}-v}\right)-2 \frac{v}{v_{\star}}\right]\right),
\end{aligned}
$$

where $v$ is the velocity of the inspiraling $\mathrm{BH}$, and $f\left(v_{\star}\right)$ the velocity distribution of field stars. The second term in parenthesis 
of Equation (4) represents the frictional force due to stars moving faster than the test mass (Chandrasekhar 1943). Such "nondominant" terms are neglected in the standard Fokker-Plank treatment in which the dynamical friction coefficient is obtained by integrating only over field stars with velocity smaller than that of the test particle (e.g., Hopman \& Alexander 2006; Alexander \& Hopman 2009; Merritt 2010). Antonini \& Merritt (2012) showed that this approximation breaks down in a shallow density profile of stars around an $\mathrm{MBH}$ where such terms can become dominant, as there are a few or even no particles moving more slowly than the local circular velocity.

The $N$-body integrations show that the stellar distribution changes with time in a quasi-self-similar way-the stellar density profile break radius shrinks progressively with time while the outer profile slope is maintained roughly unchanged. In order to account for such evolution, we computed at each time the best-fitting density model of Equation (1) to the density profile of stars in the $N$-body system at that time. We used this density model to compute gravitational potential, distribution function, and corresponding dynamical friction coefficient. This procedure allowed us to include the evolution of the stellar background when evolving the $\mathrm{BH}$ population. Our integrations are unique in the sense that they are the first ones to simultaneously include (1) a correct estimate of the dynamical friction coefficient, which takes into account the contribution of stars moving faster than the inspiraling $\mathrm{BH}$, and (2) a realistic treatment of the evolution of the stellar background under the influence of gravitational encounters. However, since our analysis does not take into account $\mathrm{BH}-\mathrm{BH}$ self-interactions, our integrations are only valid until the density of BHs remains well below the density of stars. In this respect, our approach is limited to the early evolution of the system, when the BHs only represent a negligible perturbation on the evolution of the light component.

The upper panel of Figure 4 shows the density profile of BHs obtained from the semi-analytical modeling described above and compares it with the results from the direct $N$-body simulation with the $\mathrm{BH}$ fraction $f_{\mathrm{bh}}=10^{-3}$. The central density of BHs increases with time at a rate that is comparable in the two models. The plot shows that the spatial distribution of stellar-mass BHs near the GC might not have reached a steady-state form-at least if their initial distribution was similar to what used in our models. In fact, even after a time of $\approx 8 \mathrm{Gyr}$, the central density of BHs is still substantially lower than the density of stars. As our analysis demonstrates, the persistence of such low densities of BHs is a direct consequence of the long timescale of inspiral in a density core near an $\mathrm{MBH}$. This latter point is illustrated in the lower panel of Figure 4, which shows the trajectory of a $10 M_{\odot} \mathrm{BH}$ at the GC. The rate of orbital decay slows down as the $\mathrm{BH}$ reaches $\approx r_{0} / 2$ because of the lack of low-velocity stars in the core. After the $\mathrm{BH}$ reaches this radius, dynamical friction becomes very inefficient and the decay of the $\mathrm{BH}$ orbit proceeds at a rate that is comparable to the rate at which the core radius in the stellar distribution shrinks because of gravitational encounters-a time of order the relaxation time of the nucleus.

\subsection{Comparison with Recent Work}

In this section we used direct $N$-body integrations as well as analytic models to describe the evolution of multi-mass models of the Milky Way NC characterized by an initial parsec-scale core in the density distribution. Calculations similar to those described here were recently performed by Preto \& Amaro-Seoane (2010) and Gualandris \& Merritt (2012).
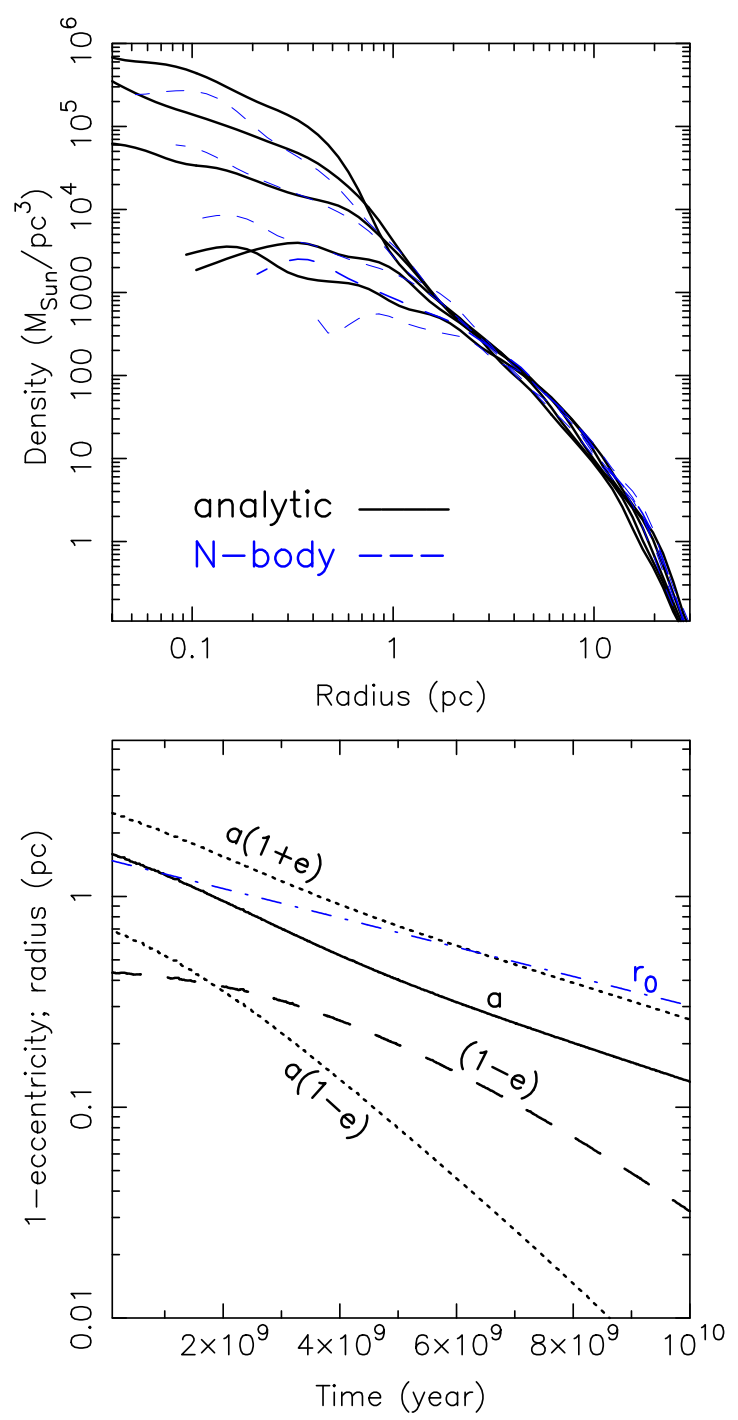

Figure 4. Top panel shows the evolution of the density of $10 M_{\odot} \mathrm{BHs}$ due to dynamical friction against the field stars. The density profiles are shown at $t=(0,2,4,6,8)$ Gyr. Blue dashed curves were obtained via direct $N$-body simulations; black solid curves correspond to the results of our semi-analytical model in which the frictional force was computed using Equation (4), which accounts for the contribution of the fast-moving stars to the frictional force. The bottom panel shows the evolution of angular momentum $(1-e)$ and semi-major axis $(a)$ of a $10 M_{\odot} \mathrm{BH}$ in our GC model. The blue dot-dashed curve shows the break radius evolution of the background model. Note that as the $\mathrm{BH}$ reaches roughly $r_{0} / 2$, its orbital radius migrates inward on a timescale similar to that over which the core in the background density evolves because of two-body relaxation.

(A color version of this figure is available in the online journal.)

Preto \& Amaro-Seoane (2010) studied the evolution of models with two mass species: stars and BHs. These authors concluded that mass segregation of the heavy component speeds up cusp growth in the lighter component by factors up to 10 in comparison with the single-mass case. This conclusion is somewhat in agreement with the results of our simulations, which also show that a stellar cusp, extending out to roughly $r_{\text {infl, }}$, regrows faster in models with BHs (e.g., Figure 2). However, for realistic numbers of $\mathrm{BHs}$ we find that the timescale of cusp regrowth is only a factor of two shorter than in the single-mass component models. Preto \& Amaro-Seoane (2010) argued that the timescales associated with cusp regrowth are clearly shorter than the Hubble time for nuclei similar to that of the Milky 
Way-even though the relaxation time, as estimated for a singlemass stellar distribution, exceeds one Hubble time. On the basis of our study, we conclude instead that over one Hubble time, and if a standard IMF is adopted, adding a heavy component has relatively little effect on the evolution of the main-sequence component (e.g., Figure 3). Even for a top-heavy IMF, which results in initial larger densities of $\mathrm{BHs}$, the time for cusp regrowth is longer than the mean stellar age in the Galactic center ( $\sim 5$ Gyr). The reason for this is that because of the inefficient dynamical friction force in a density core around an $\mathrm{MBH}$, the central density of BHs remains well below the density of stars for a time of order the relaxation time of the nucleus. The time required to regrow a cusp in the stellar distribution appears to be longer than the Hubble time for galaxies similar to the Milky Way.

Gualandris \& Merritt (2012) simulated the merger between galaxies with MBHs containing four mass groups, representative of old stellar populations. They followed the evolution of the merger products for about three relaxation times and found that the density cores formed during the galaxy mergers persisted and that the distribution of the stellar-mass black holes evolved "against an essentially fixed stellar background." Gualandris \& Merritt (2012) also integrated the exact same Fokker-Planck models as in Preto \& Amaro-Seoane (2010) and argued that the accelerated cusp growth described by these latter authors is seen to be present only at small radii, $r \lesssim 0.05 r_{\text {infl. }}$. At radii larger than these, adding the BHs has the effect of lowering the density of the stellar component at all times. Gualandris \& Merritt (2012) argued that Preto \& Amaro-Seoane (2010) were misled by looking at the very-small-radius regime in their Fokker-Plank solutions, where the cusp in the main-sequence component stands out. Our study shows that the $\mathrm{BH}$ population has indeed two effects on the main-sequence population: it lowers the "mean" density of stars (the point stressed in Gualandris \& Merritt 2012), and it accelerates the redistribution of the stars in the phase space toward the $\gamma=3 / 2$ steady state (as found in Preto \& Amaro-Seoane 2010). So in a sense, the BHs both "create" and "destroy" a cusp: although the presence of a $\mathrm{BH}$ population can significantly accelerate the timescale of cusp regrowth in the stellar distribution, the scattering off the heavy (BH) component causes the density of stars to decrease at radii larger than $\sim 0.05 r_{\text {infl }}$.

\section{GLOBULAR CLUSTER MERGER MODEL_EVOLUTION TIMESCALES}

In the previous section we have shown that because of the long timescales of evolution, the current distribution of BHs and stars at the center of galaxies similar to the Milky Way should be considered very uncertain. In these and more massive galaxies, the current distribution of stars and BHs can still reflect their initial conditions and the processes that have led to the formation and evolution of their central NC. This conclusion suggests that standard mass-segregation models, which assume the same initial phase space distribution for BHs and stars, can lead to misleading conclusions regarding the current dynamical state of galactic nuclei and motivates studies that start from initial conditions that correspond to well-defined physical models.

In what follows, we present a set of $N$-body experiments that were designed to understand the distribution of stars and $\mathrm{BHs}$ in galactic nuclei formed via repeated merger of massive stellar clusters - a formation model that has been shown to be very successful in reproducing the observed properties and scaling relations of nuclear star clusters (e.g., Turner et al. 2012; Antonini 2013). We begin here with discussing the relevant timescales of the problem, including the characteristic orbital decay time of massive clusters in the inner regions of galaxies, and the relaxation timescales of galactic nuclei and globular clusters.

\subsection{Globular Clusters Decay Time}

Sufficiently massive and compact clusters can decay toward their parent galaxy central region in a time much shorter than the Hubble time. An approximation of the time for clusters (within the half mass radius of the stellar bulge) to spiral to the center is given by (Antonini et al. 2012):

$$
\Delta t_{h} \approx 3 \times 10^{10} \mathrm{yr} \tilde{R}_{\mathrm{eff}}^{1.75} \tilde{m}^{-1}
$$

where $\tilde{R}_{\text {eff }}$ is the galactic effective radius in kiloparsecs, and $\tilde{m}$ is the globular cluster mass in units of $10^{6} M_{\odot}$. Within $\Delta t_{h}$ the forming nucleus has a luminosity comparable to that of the surviving clusters. Equation (5) predicts that a significant fraction of the globular cluster population in faint and intermediate luminosity stellar spheroids would have spiraled to the center by now, while in giant ellipticals, because of their larger characteristic radii, the time required to grow an $\mathrm{NC}$ might be longer than $10^{10} \mathrm{yr}$. We stress that the inspiral time obtained using Equation (5) gives only a crude approximation (likely an overestimate) of the real dynamical friction timescale. Nevertheless, it is reasonable to draw the conclusion that the observed lack of nuclei in galaxies more massive than about $10^{10} M_{\odot}$ could be due to the longer infall times in these galaxies, due to their larger values of $R_{\text {eff }}$. Figure 5 presents a test of this idea. This figure gives effective radii versus masses, $M_{\text {gal }}$, for galaxies belonging to the Virgo cluster that either have (filled black circles) or do not have (star symbols) a central NC. Dashed curves give the value of $R_{\text {eff }}$ obtained by setting $\Delta t_{h}=10^{10} \mathrm{yr}$ in Equation (5) and adopting various masses of the sinking object. The figure shows that only in galaxies with $M_{\text {gal }} \lesssim 10^{10} M_{\odot}$, massive globular clusters would have enough time to spiral into the center, merge, and form a compact nucleus. The observed absence of compact nuclei in giant ellipticals could be therefore interpreted as a consequence of the long dynamical friction timescale of globular clusters in these galaxies.

We add that the density profile of stars in giant ellipticals is often observed to be flat or slowly rising inside the influence radius of the $\mathrm{MBH}$. As shown in Section 2 this implies a very long dynamical friction timescale inside the $\mathrm{MBH}$ influence radius due to the absence of stars moving more slowly than the local circular velocity (Antonini \& Merritt 2012). Massive clusters orbiting within the core of a giant elliptical galaxy do not reach the center even after $10^{10} \mathrm{yr}$. In addition, because of the strong tidal field produced by the $\mathrm{MBH}$, globular clusters can only transport little mass to the very central region of the galaxy. Both these effects, i.e., long inspiral times and little mass transported to the center, have been suggested to suppress the formation of $\mathrm{NCs}$ in bright galaxies, in agreement with observations (Antonini 2013).

\subsection{Nuclear Star Clusters Relaxation Time}

A useful reference time for our study is the relaxation time computed at the radius containing half of the mass of the system, $R$. Setting $\ln \Lambda=12$, and ignoring the possible presence of an 




Figure 5. Effective radii ( $\left.R_{\text {eff }}\right)$ of galaxies plotted against their masses $\left(M_{\mathrm{gal}}\right)$. Filled circles and star symbols represent, respectively, nucleated and unnucleated galaxies that belong to the Virgo galaxy cluster (Côté et al. 2006). Dots are data from Forbes et al. (2008). The vertical line gives the value of the mass $\left(M_{\text {gal }} \approx 10^{10.5} M_{\odot}\right)$, which approximately set the transition between nucleated galaxies $\left(M_{\mathrm{gal}}<10^{10.5} M_{\odot}\right)$, and MBH dominated galaxies $\left(M_{\text {gal }}>10^{10.5} M_{\odot}\right)$ (Côté et al. 2006). Dashed horizontal curves give the value of $R_{\text {eff }}$ obtained by setting $\Delta t_{h}=10^{10}$ in Equation (5) and adopting various masses of the sinking objects. The large effective radii of massive galaxies give timescales for inspiral that are usually longer than one Hubble time. The observed lack of nuclei in massive galaxies could be explained as a consequence of the longer infall times in these galaxies, due to their large effective radii.

$\mathrm{MBH}$, the half mass relaxation time is

$$
T_{h}=2.1 \times 10^{5} \frac{\left[r_{h}(\mathrm{pc})\right]^{3 / 2} \mathrm{~N}^{1 / 2}}{\left(\mathrm{~m} / M_{\odot}\right)^{1 / 2}} \frac{10}{\ln (0.4 N)} \mathrm{yr},
$$

where $N$ is the total number of stars.

In the absence of an $\mathrm{MBH}$, collisional relaxation leads to mass segregation and core collapse. In a preexisting $\mathrm{NC}$, the presence of an MBH inhibits core collapse, causing instead the formation of a Bahcall-Wolf cusp, $n \sim r^{-7 / 4}$, on the two-body relaxation timescale (Preto et al. 2004; Merritt 2009). Nuclear clusters belonging to the Virgo galaxy cluster have a half-mass relaxation time that scales with the total absolute magnitude of the host galaxy, $M_{B}$, as (Merritt 2009):

$$
\log \left(T_{h} / \mathrm{yr}\right)=9.38-0.43\left(M_{B}+16\right) .
$$

Galaxies with luminosities less than $\sim 4 \times 10^{8} L_{\odot}$ have NCs with relaxation times that fall below $10 \mathrm{Gyr}$. These galaxies have NCs with masses $\lesssim 10^{7} M_{\odot}$ and half mass radii $r_{h} \lesssim 10 \mathrm{pc}$. These limiting values appear close to those characterizing the Milky Way NC, suggesting that only spheroids fainter than the Milky Way have collisionally relaxed nuclei. Relaxation times for nuclei with masses $\gtrsim 10^{7} M_{\odot}$ are therefore too long for assuming that they have reached a collisionally relaxed state, but they are still short enough that gravitational encounters would substantially affect their structure over the Hubble time. This is in agreement with the results of Section 2 and also appears to be consistent with absence of a Bahcall-Wolf cusp in the distribution of stars at the GC.

\subsection{Globular Clusters Relaxation Time}

Globular clusters with $N \sim 10^{6-7}$ have relaxation times $T_{h} \sim 10^{9-10}$ yr. Most Galactic globular clusters are therefore relaxed systems. The timescale required for the $\mathrm{BH}$ population to segregate to the cluster center and form a subcluster dynamically decoupled from the host stellar cluster is approximately the core-collapse time for the initial BH cluster (e.g., Banerjee et al. 2010):

$$
T_{m s} \approx \frac{m}{m_{b h}} T_{c c}
$$

where $m_{b h}$ is the mass of a stellar black hole, and $T_{c c}$ is the core-collapse time of the host stellar cluster, which is about $T_{c c} \approx 15 \times T_{h}$ for a Plummer model. After $\sim T_{m s}$ the central density of BHs becomes large enough that $\mathrm{BH}-\mathrm{BH}$ binary formation takes place through three or four body interactions (Heggie \& Hut 2003). The formed BH binaries then "harden" through repeated superelastic encounters that lead to the ejection of BHs from the cluster core until eventually only a few BHs are left (e.g., Banerjee et al. 2010).

In galaxies similar to the Milky Way, stellar clusters with masses $\gtrsim 10^{6} M_{\odot}$ and starting from a galactocentric radius of $1 \mathrm{kpc}$ have orbital decay times due to dynamical friction less than $\lesssim 3$ Gyr (Equation (5)). The cluster's dynamical friction time is therefore typically long compared with the timescale over which the BHs would segregate to the center of the cluster.

It is possible that the $\mathrm{BH}$ population will evaporate through superelastic encounters before the cluster reaches the center of the galaxy. This could lead to the formation of an NC with a much smaller abundance of BHs relative to stars than what is predicted by standard initial mass functions. On the other hand, for massive clusters after the BHs are already segregated to the center, the encounter driven evaporation timescale of the $\mathrm{BH}$ sub-cluster typically requires an additional few Gyr of evolution to complete (Dowing et al. 2010, 2011). Moreover, recent theoretical studies (Morscher et al. 2013; Sippel \& Hurley 2013), together with several observational evidences (Maccarone et al. 2007, 2011; Brassington et al. 2010; Strader et al. 2012), show that old globular clusters may still contain hundreds of stellar $\mathrm{BHs}$ at present, which suggests that $\mathrm{BH}$ depletion might not be as efficient as previously thought. This indicates that for many large clusters (the ones most relevant to NC formation), most of the BHs will not be ejected before inspiral has occurred. The above arguments convinced us that the inspiral of massive clusters in the central region of the Galaxy could serve as a continuous source term of $\mathrm{BHs}$ in these regions.

\section{NUMERICAL SETUP}

\subsection{Initial Conditions and Numerical Method}

In Antonini et al. (2012) we used $N$-body simulations to study how the presence of an MBH at the center of the Milky Way impacts the globular cluster merger hypothesis for the formation of its NC. We determined the properties of the stellar distribution in a galactic nucleus forming through the infall and merging of globular clusters. We showed that a model in which a large fraction of the mass of the Milky Way NC arose from infalling globular clusters is consistent with existing observational constraints. Here we replaced the single-mass globular cluster models of Antonini et al. (2012) with systems containing (in addition to the stellar component) a remnant population of BHs. 
Table 1

Initial Models

\begin{tabular}{lcrccc}
\hline \hline Run & \multicolumn{1}{c}{$N_{\text {gal }}$} & $N_{\mathrm{cl}, \star}$ & $N_{\mathrm{cl}, \mathrm{bh}}$ & No. of Infalls & Galaxy Model \\
\hline A1 & $1 \times 10^{6}$ & 45720 & 240 & 1 & Model 1 \\
A2 & $1.5 \times 10^{6}$ & 45720 & 240 & 1 & Model 2 \\
B & $4.6 \times 10^{5}$ & 5715 & 33 & 12 & Model 1 \\
C & $4.6 \times 10^{5}$ & 5715 & 100 & 12 & Model 1 \\
\hline
\end{tabular}

These simulations were performed by using $\phi$ GRAPE (Harfst et al. 2006), a direct-summation code optimized for running on GRAPE accelerators (Makino \& Taiji 1998). This integrator is equivalent to $\phi$ GRAPEch, which we used in Section 2, but without the regularized chain. The accuracy and performance of the code are set by the time-step parameter $\eta$ and the softening length $\epsilon$. We used a Plummer softening for the gravitational force between particles, and we did not model binary formation in the calculation reported below. We set $\eta=0.01$ and $\epsilon=10^{-4} \mathrm{pc}$. With these choices, energy conservation was typically $\lesssim 0.01 \%$ during each merging event. The simulations were carried out using the 32 node GRAPE cluster at the Rochester Institute of Technology and also on Tesla C2050 graphics processing units on the Sunnyvale cluster at the Canadian Institute for Theoretical Astrophysics. In the latter integrations, $\phi \mathrm{GRAPE}$ was used in serial mode with SAPPORO (Gaburov et al. 2009). Each simulation required between three and four months total of computational time.

Table 1 summarizes the parameters of the $N$-body models. We performed four simulations. Runs A1 and A2 are highresolution simulations $\left(N \sim 10^{6}\right)$ that explore the dynamics of one single globular cluster inspiral. In simulations B and $\mathrm{C}$, the total number of $\mathrm{N}$-body particles was greatly reduced in order to more efficiently follow the consecutive inspiral and merger of 12 dynamically evolved clusters. In these latter simulations, each inspiral simulation was started after the stars from the previously disrupted cluster were set to a state of collisionless dynamical equilibrium and the number of $\mathrm{BHs}$ in the cluster remnant dropped to $<10$. This corresponds to a time of $1-3 \times 10^{7} \mathrm{yr}$ for each inspiral event to complete, with the longer times corresponding to the earlier infalling clusters. The clusters were initially placed on circular orbits at a distance of $20 \mathrm{pc}$ from the center. The choice of circular orbits was motivated by the well-known effect of orbital circularization due to dynamical friction (e.g., Casertano et al. 1987; Hashimoto et al. 2003). In the consecutive merger simulations (runs B and C), in order not to favor any particular direction for the inspiral, the orbital angular momenta were selected in the following way: the surface of a sphere can be tessellated by means of 12 regular pentagons, the centers of which form a regular dodecahedron inscribed in the sphere. The coordinates of the centers of these pentagons were identified with the tips of the 12 orbital angular momentum vectors. In this way, the inclination and longitude of ascending node of each initial orbit were determined.

\subsection{Galaxy Models}

We adopted two different $N$-body models to represent the central region of the Galaxy. Model 1 is obtained by an inner extrapolation of the observed density profile of stars in the Galactic nuclear bulge outside $10 \mathrm{pc}$. In these regions the Galaxy is dominated by the presence of the nuclear stellar disk, which is characterized by a flat density profile. Accordingly, we adopted the truncated shallow power-law density model:

$$
\rho_{\mathrm{gx}, 1}(r)=\tilde{\rho}\left(\frac{r}{\tilde{r}}\right)^{-\gamma} \operatorname{sech}\left(\frac{r}{r_{\mathrm{cut}}}\right),
$$

where $\tilde{\rho}=400 M_{\odot} \mathrm{pc}^{-3}$ is the density at $\tilde{r}=10 \mathrm{pc}$, and the truncation radius is $r_{\text {cut }}=20 \mathrm{pc}$. Hence, the initial conditions for model 1 do not include a preexisting $\mathrm{NC}$, and they correspond to a shallow density cusp around a central $\mathrm{MBH}$.

Model 2 was obtained by the superposition of the density model of Equation (9) and a broken power-law model representing an NC:

$$
\begin{aligned}
\rho_{\mathrm{gx}, 2}(r)= & \rho_{\mathrm{gx}, 1}(r)+\rho_{b}\left(\frac{r}{r_{b}}\right)^{-\gamma_{i}} \\
& \times\left[1+\left(\frac{r}{r_{b}}\right)^{\alpha}\right]^{\left(\gamma_{i}-\beta\right) / \alpha} \operatorname{sech}\left(\frac{r}{r_{\text {cut }}}\right),
\end{aligned}
$$

with $\rho_{b}=4.1 \times 10^{4} M_{\odot} \mathrm{pc}^{-3}, r_{b}=1.5 \mathrm{pc}, \gamma_{i}=0.5$, $\beta=1.9, \alpha=3.73$, and $r_{\text {cut }}=20$ pc. This model corresponds approximately to the best-fitting density profile of the simulation end-products of Antonini et al. (2012).

In both model 1 and model 2 we included a central $\mathrm{MBH}$ of mass $M_{\bullet}=4 \times 10^{6} M_{\odot}$ and we generated their $N$-body representations via numerically calculated distribution functions.

\subsection{Star Clusters Model}

We generated our globular cluster initial conditions following the same procedure described in Antonini et al. (2012), where a detailed description of the initial conditions of the clusters can be found. In brief, the clusters are started on circular orbits of radius $r=20 \mathrm{pc}$, and their initial masses and radii are set up in such a way as to be consistent with the galactic tidal field at that radius. The clusters are King models (King 1962) with central (King) potential $W_{0}=5.8$, core radius $r_{k}=0.5 \mathrm{pc}$, and central velocity dispersion $\sigma_{K}=35 \mathrm{~km} \mathrm{~s}^{-1}$. With this set of parameters the truncated mass of the clusters was $m_{\mathrm{cl}} \approx 1.1 \times 10^{6} M_{\odot}$.

To these models we added a heavier mass group representing a population of stellar BHs. The relative values of the particle masses was 1:10. These represent one solar mass stars and $10 M_{\odot}$ BHs, respectively. The two mass groups had the same initial phase-space distribution. Standard population synthesis models predict that about $1 \%$ of the total mass in a stellar system will be in BHs, top-heavy mass functions result in about five times more BHs. Accordingly, in our initial models the total mass in BHs was $10^{-2}$ and $5 \times 10^{-2}$ times $4 \times 10^{6} M_{\odot}$ (the mass of the non-truncated King model) for runs $\mathrm{A}$ and $\mathrm{B}$ and run $\mathrm{C}$ (Table 1), respectively. The choice of scaling the total number of BHs to the initial non-truncated cluster mass is based on the fact that when the cluster reaches $100 \mathrm{pc}$ (roughly the radius at which our models start to be tidally truncated) mass segregation is likely to have already occurred. under these circumstances, tidal stripping will preferentially remove stars from the outer part of the system, leading to an overabundance of BHs with respect to standard mass functions (Banerjee \& Kroupa 2011). In addition, this choice resulted in a sufficiently good statistics for the remnants population. The effect of varying the initial mass in stellar BHs and their initial dynamical state inside the clusters will be investigated in a future paper.

The mass-segregated cluster models were then created via $N$-body integrations, starting from the cluster equilibrium models. Figure 6 gives the evolution of the two mass components 

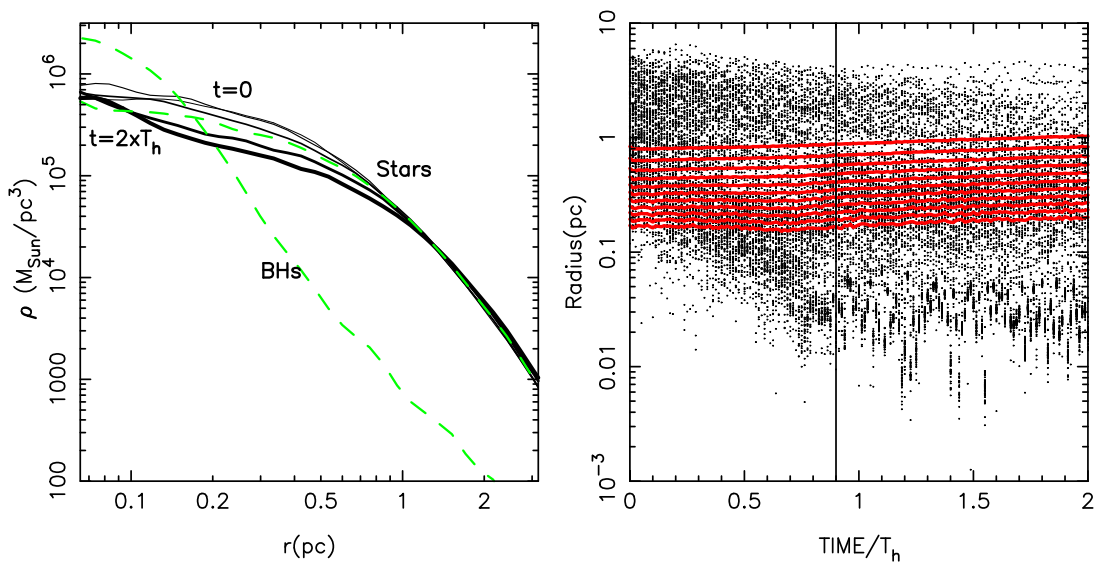

Figure 6. Evolution of the cluster model during the initial $N$-body integrations used to realize the mass-segregated cluster models for runs A1 and A2 (see Table 1). The left panel shows the evolution of the stellar density profile. The line thickness increases with time. The green dashed curves give the density profile of stars and BHs used as initial conditions for the inspiral runs. The right panel gives the Lagrangian radii of the stellar component (red solid curves) and the BHs distance from the cluster center (black dots). The BHs segregate to the cluster core, forming a dense sub-cluster in about one half-mass relaxation time as defined by the stellar component. The stellar cluster slightly expands because of heating by the inspiraling BHs. The vertical line gives the time at which we extracted the initial conditions for the inspiral simulations.

(A color version of this figure is available in the online journal.)

during these integrations. We let the system evolve for a few relaxation times as defined by Equation (7). The stellar BHs accumulate toward the center and by approximately one half-mass relaxation times their distribution appears to have reached an approximately steady state. At the same time the density profile defined by the stellar component undergoes a slow expansion due to heating by the BHs.

\section{RESULTS}

\subsection{Single Inspiral Simulations}

Figure 7 shows surface density contours of the single inspiral simulation A1. After $\sim 10^{7} \mathrm{yr}$ the stellar cluster is at about $5 \mathrm{pc}$ from the center; at these distances the disruption process due to tidal stress from the central MBH begins. Rapid removal of stars from the outer part of the cluster by the galaxy and MBH tidal fields unveils its mass-segregated BH cluster. Figure 8 shows the time evolution of radius and bound mass for the globular clusters in runs A1 and A2. Our cluster models rapidly evolve to a state of dark stellar cluster, i.e., a dense cluster dominated by dark stellar remnants (Banerjee \& Kroupa 2011). After this state is reached, because of the drop in the total cluster mass (see lower panels of Figure 8), the dynamical friction drag on the remaining $\mathrm{BH}$ cluster is largely suppressed, slowing down its orbital decay toward the center of the galaxy. Noticeable, because of the common motion around the system $\mathrm{MBH}$-cluster center of mass, the $\mathrm{MBH}$ is significantly displaced from the galaxy center. More precisely, we found a maximum displacement of $\sim 5 \mathrm{pc}$ in run A1 and a somewhat smaller (maximum) displacement of $\sim 2 \mathrm{pc}$ in run $\mathrm{A} 2$.

Figure 8 shows that after the stellar clusters are disrupted, the remaining dark clusters have a bound mass of $\sim 2.7 \times$ $10^{3} M_{\odot}$ in simulation $\mathrm{A} 1$ and $1.7 \times 10^{4} M_{\odot}$ in simulation A2, corresponding to 9 and $67 \mathrm{BH}$ particles, respectively. The enhanced removal of stars and BHs decelerates the orbital evolution of the cluster because of its lower mass. During the inspiral after about $2 \times 10^{7} \mathrm{yr}$ the $\mathrm{BH}$ cluster core has collapsed to $\lesssim 0.05 \mathrm{pc}$. This makes the central density much higher, which prevents the complete disruption of the $\mathrm{BH}$ cluster.

Assume that the cluster has reached a state of "thermal equilibrium" at the center, i.e., a state in which the stars and BHs are represented by lowered Maxwellians: $m_{\mathrm{st}} \sigma_{\mathrm{st}}^{2}=$ $m_{\mathrm{bh}} \sigma_{\mathrm{bh}}^{2}$, where $\sigma_{\mathrm{st}}\left(\sigma_{\mathrm{bh}}\right)$ is the central one-dimensional velocity dispersion of the stars (BHs). If an $\mathrm{MBH}$ of mass $M_{\bullet}$ is present at the center of the galaxy, disruption occurs at distance

$$
r_{\mathrm{disr}} \approx 2\left(\frac{\sigma_{\mathrm{NC}}}{5 \sigma_{K}}\right)^{2 / 3}\left(\frac{r_{\text {infl }}}{r_{K}}\right)^{1 / 3} r_{K}
$$

from the $\mathrm{MBH}$. Then the $\mathrm{BH}$ cluster tidal disruption radius will be smaller than that of the stellar cluster by a factor

$$
\approx 0.3\left(20 \frac{r_{K ; \mathrm{bh}}}{r_{K ; \mathrm{st}}}\right)^{2 / 3}\left(\frac{1}{10} \frac{m_{\mathrm{bh}}}{m_{\mathrm{st}}}\right)^{1 / 3}
$$

which suggests that the BHs can end up being more centrally concentrated than the stars. A condition for this to happen is that the $\mathrm{BH}$ cluster must not evaporate before it has lost significant orbital energy by dynamical friction. In fact, the internal evolution of a compact $\mathrm{BH}$ cluster embedded in the extreme tidal field of the GC can proceed very rapidly and lead to the cluster complete dissolution on a short timescale, of order a few Myr (e.g., Banerjee \& Kroupa 2011; Gürkan $\&$ Rasio 2005). In our simulations the internal dynamical evolution of the clusters has been suppressed by giving a nonnegligible softening radius to the cluster particles. In fact, the adopted integrator cannot treat the postcollapse evolution of the cluster, since we used a softened potential. Thus, we terminated the simulation at $\approx 3 \times 10^{7} \mathrm{yr}$ of evolution after the clusters have been fully depleted of stars and the remaining $\mathrm{BH}$ clusters underwent core collapse.

The end-product spatial density profile and cumulative mass distribution of stars and BHs are given in Figure 9. In order to obtain the $\mathrm{BH}$ density profile, we forced the unbinding of the remnant clusters after $10^{7} \mathrm{yr}$ of evolution. The unbinding of the clusters was induced by "turning off" the gravitational interaction terms between the BH cluster members and by letting the system evolve for about one crossing time. Although quite artificial, this procedure allowed us to account for the fact that the dissolution time of the cluster remnants is expected to be short relative to their dynamical friction timescale-in our models a $10^{4} M_{\odot}$ system starting from a galactocentric radius of $1 \mathrm{pc}$ 

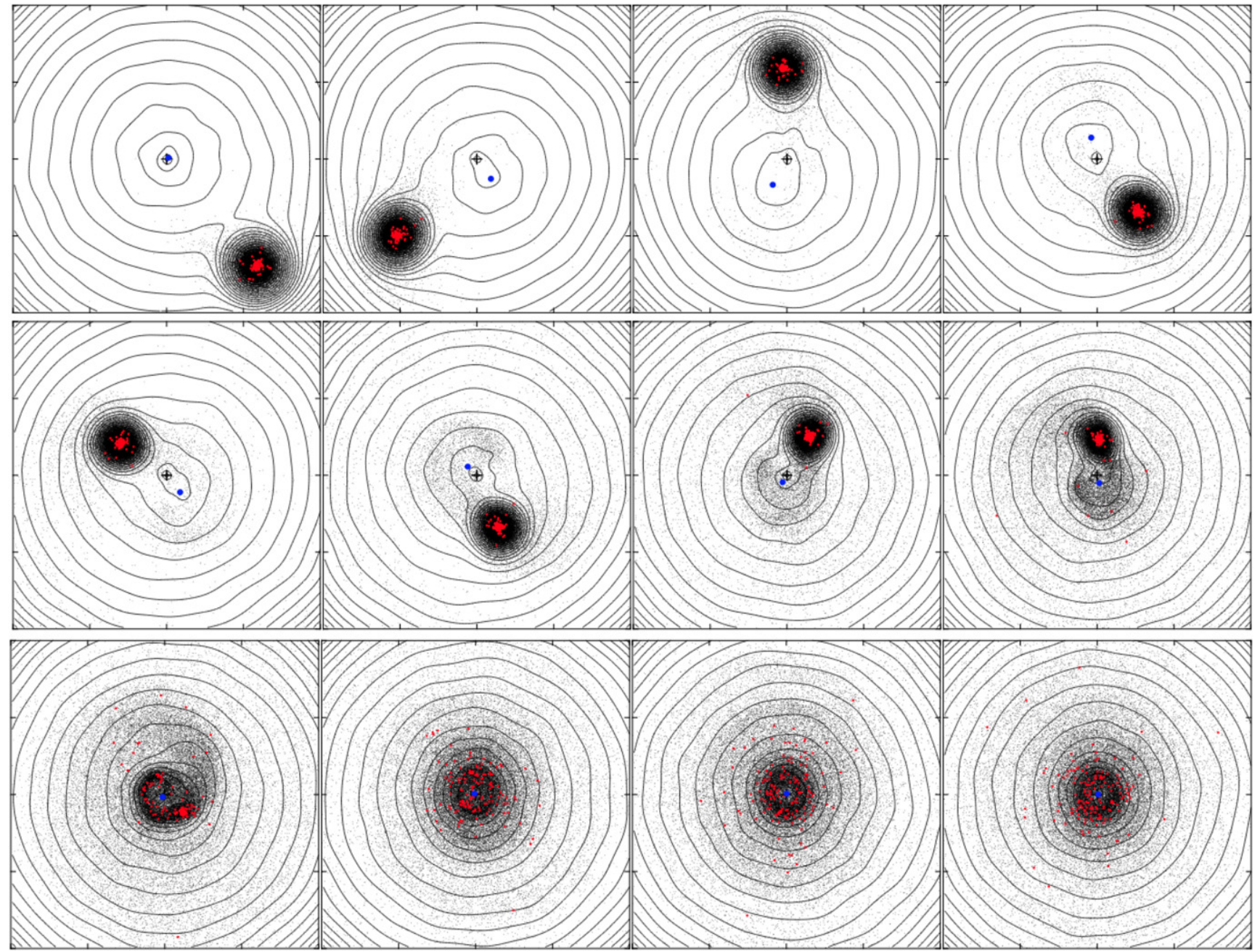

Figure 7. Inspiral of a $\sim 10^{6} M_{\odot}$ globular cluster in a Milky Way model. The linear size of each box is $20 \mathrm{pc}$; the time separation between each snapshot is $5 \times 10^{5}$ yr. The blue filled circle marks the galactic MBH position. Curves show the contours of the projected density of the background galaxy. Red points represent the globular cluster BHs; black dots the cluster stars.

(A color version of this figure is available in the online journal.)

reaches a radius of $0.2 \mathrm{pc}$ after $10^{8} \mathrm{yr}$. The $\mathrm{BH}$ clusters will dissolve on a timescale proportional to the half-mass relaxation time, $T_{e v} \approx 300 \times T_{h}$ (Spitzer 1987) (if we ignore the effect of the external tidal field); from Equation (6), using $r_{h}=0.1 \mathrm{pc}$ (Figure 6), $N=1000$, and $m=10 M_{\odot}$ we find $T_{e v} \approx 10^{7} \mathrm{yr}$. We stress that since the current state of the art computational capability does not allow us to simulate the actual number and mass of stars and to calculate the internal evolution of the cluster, the densities of BHs obtained here should be only considered as approximate (likely an underestimate of the real density). Note also that it is unlikely that the core-collapse phase of a BH cluster can lead to the formation of an intermediate mass black hole since any $\mathrm{BH}-\mathrm{BH}$ merger will eject the remnant from the cluster via asymmetric emission of gravitational wave radiation before it can accrete other BHs or surrounding stars.

The number of BHs in our simulations, $N_{\mathrm{BH}, \mathrm{nb}}(<r)$, was converted to a predicted number for a Milky Way model, using the approximate scaling

$$
N_{\mathrm{bh}}(<r)=N_{\mathrm{BH}, \mathrm{nb}}(<r) \times \frac{4 \times 10^{6} M_{\odot}}{10 M_{\odot}} \frac{m_{\mathrm{BH}, \mathrm{nb}}}{M_{\bullet}},
$$

with the last factor of the second term containing the masses in the units of the $N$-body code. The number of BHs transported in the inner $\sim 1 \mathrm{pc}$ is of order 100 in both $\mathrm{A} 1$ and $\mathrm{A} 2$. Our simulations result in a mass distribution characterized by a flat density core inside $\sim 2 \mathrm{pc}$ in run $\mathrm{A} 1$ and $\sim 3 \mathrm{pc}$ in run $\mathrm{A} 2$ and an envelope that falls off rapidly at large radii. The $\mathrm{BH}$ density distributions flatten within a radius comparable to the size of the core observed in the stellar density profile. This is because the BH cluster does not experience significant orbital decay after the star cluster is fully dispersed. The difference in the mass distribution in the two models A1 and A2 is caused by the difference in the enclosed mass in the background galaxy. The tidal field near the galactic center is much stronger in simulation A2 because of the presence of a preexisting compact stellar nucleus. The stronger tidal field in the galaxy model of simulation A2 results in a larger core in the density distribution due to the larger tidal disruption radius of the star cluster.

The dashed curves in Figure 9 display the density profile of the galaxy background at the end of the simulations. A comparison with the functional forms of Equations (9) and (10) used to generate the initial equilibrium models shows that the background galaxy in A1 did not evolve appreciably; in A2, instead, the final density of the galaxy appears to have slightly changed, showing higher central densities and a smaller core radius $(\sim 0.5 \mathrm{pc})$ than the initial model. Thus, the influence of the inspirals on the preexisiting distribution of field stars in 

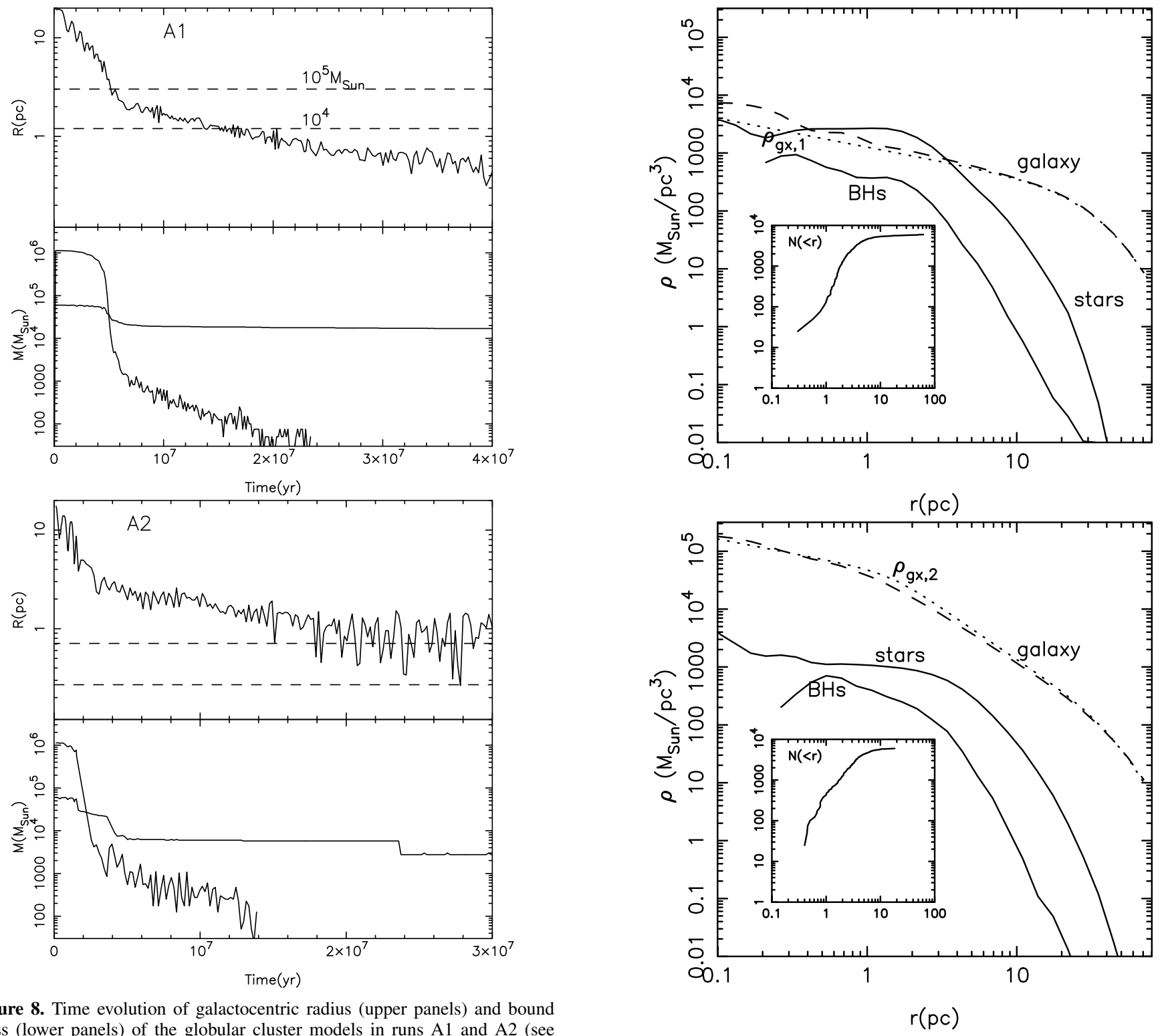

Figure 8. Time evolution of galactocentric radius (upper panels) and bound mass (lower panels) of the globular cluster models in runs A1 and A2 (see Table 1). In the lower panels we show separately the bound mass in BHs (curve starting at $6 \times 10^{4} M_{\odot}$ ) and in stars (curve starting at $1.1 \times 10^{6} M_{\odot}$ ) Dashed curves in the upper panels give the galactocentric radii in the initial galaxy models containing $10^{4}$ and $10^{5} M_{\odot}$.

our models is negligible at large radii $\gtrsim 3 \mathrm{pc}$, while it leads to slightly higher central densities within this radius and a smaller core radius relative to the initial model distribution (we discuss this point in more detail below in Section 5.3).

The key question to be answered by these simulations is the degree to which the density of stellar BHs near the center of the galaxy is enhanced, after the inspiral, with respect to the relative density expected in the absence of dynamical evolution. Figure 10 shows the radial profile of the ratio, $\rho_{\mathrm{bh}} / \rho_{\mathrm{st}}$, of $\mathrm{BHs}$ to stellar densities. The dotted curve in the figure gives the density ratio, $\rho_{\mathrm{bh}} / \rho_{\mathrm{st}}=0.052$, of the initial model before the $\mathrm{BHs}$ segregate to the cluster center, which is also the relative $\mathrm{BH}$ density expected in the absence of the cluster dynamical evolution. Inside $5 \mathrm{pc}$, our simulations result in larger $\mathrm{BH}$ densities relative to what is expected if the remnant population had the same density distribution of stars at the moment the clusters reach their tidal disruption radius.

Figure 9. Density profile of BHs and stars at the end of the single inspiral simulations of Table 1. Upper panel corresponds to simulation A1 and lower panel to simulation A2. Density profiles are given after the $\mathrm{BH}$ cluster are artificially dispersed as described in the text. Insert panels show the corresponding cumulative number distributions of stellar BHs. Dotted curves give the density model of the initial galaxy models of Equations (9) and (10). The density profile of the galaxy at the end of the simulation is shown as dashed curves. Up to $\sim 100 \mathrm{BHs}$ are migrated inside the inner $0.1-0.2 \mathrm{pc}$ of the galactic center.

Figure 10 suggests that the evolution of an $\mathrm{NC}$ formed through the merger of dynamically evolved stellar clusters will be dominated by the BHs. The condition that the evolution of the light component is dominated by scattering off the heavy component is (e.g., Gualandris \& Merritt 2012)

$$
\rho_{\mathrm{bh}} \gtrsim\left(m_{\mathrm{st}} / m_{\mathrm{bh}}\right) \rho_{\mathrm{st}}=0.1 \rho_{\mathrm{st}} .
$$

From Figure 10 we see that the evolution of the stars will be dominated by gravitational interactions with the $\mathrm{BHs}$ - as opposed to self-interactions-within a radius of size $\sim 3 \mathrm{pc}$, roughly the $\mathrm{MBH}$ influence radius. We conclude that the postinfall long-term evolution of the systems presented here will be very different from that of the models discussed in Section 2 for 


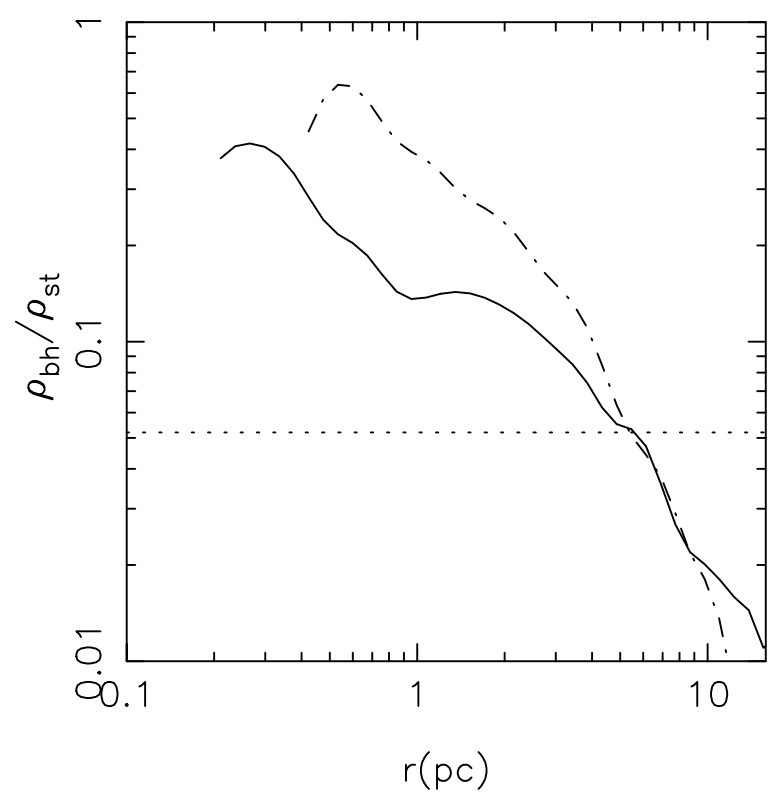

Figure 10. Radial profile of the ratio between the density of $\mathrm{BHs}, \rho_{\mathrm{bh}}$, and stellar densities, $\rho_{\text {st }}$, for simulations A1 (continue curve) and A2 (dot-dashed curve). The dotted curve shows the value corresponding to the initial cluster models before the BHs segregated to the center. The latter is also the density ratio expected in the absence of dynamical evolution. Within 5 pc our models predict larger $\mathrm{BH}$ densities than what is expected if the remnant population had the same density distribution of stars at the time the clusters reach their tidal disruption radius. As discussed in the text, where $\rho_{\text {bh }} \gtrsim 0.1 \rho_{\text {st }}$, the evolution of the stars is expected to be dominated by gravitational interaction with the $\mathrm{BHs}$, as opposed to self-interactions.

which about one Hubble time was required in order to first met the condition Equation (14).

\subsection{Consecutive Inspirals}

In order to determine the distribution of stars and stellar remnants predicted by a dissipationless formation model for the Milky Way NC, we performed simulations that followed the repeated merger of mass-segregated massive clusters in the GC; these correspond to simulations $B$ and $C$ of Table 1. In these integrations the number of $N$-body particles was much reduced with respect to the single inspiral simulations presented in Section 5.1. The sub-sampling was a necessary compromise to keep the computational time from becoming excessively long, while still allowing the simulations to follow the successive inspiral of 12 clusters into the center of the galaxy, giving a total accumulated mass in stars of $\approx 10^{7} M_{\odot}$, which is roughly the observed mass of the Milky Way NC.

The process of NC formation is illustrated in Figure 11, which shows the growing central density of stars and BHs during the globular cluster inspirals. As clusters merge to the center, the peak density of the model increases and an NC forms, appearing within the model inner $\approx 10 \mathrm{pc}$ as an excess density of stars over the background density of the galaxy. Insert panels give the radial dependence of the density profile slope of the final NC models. In the radial range $0.5 \mathrm{pc} \lesssim r \lesssim 5 \mathrm{pc}$, the spatial density profile of the merger product is characterized by a density of stars that rises steeply toward the center roughly as $\sim r^{-1.5}$. At smaller radii at the end of both simulations $\mathrm{B}$ and $\mathrm{C}$, the spatial density profile of stars flattens and the radial dependence becomes approximately $\mathrm{d} \log \rho / \mathrm{d} \log r \approx$ -1 inside $0.3-0.5 \mathrm{pc}$. The $\mathrm{BH}$ population exhibits a very steep density $\operatorname{cusp} \mathrm{d} \log \rho / \mathrm{d} \log r \approx-2.2$, outside $\sim 0.5 \mathrm{pc}$, and a somewhat flat profile within this radius. The merger process produces an NC that is in an state of advanced mass segregation, with the heavy component dominating the density of stars inside a radius of roughly $0.3 \mathrm{pc}$ and $1 \mathrm{pc}$ in simulations $\mathrm{B}$ and $\mathrm{C}$, respectively. Since smaller systems have shorter relaxation times and undergo mass segregation more quickly, the merger process effectively reduces the mass-segregation timescale of the NC compared for instance with the models discussed in Section 2.

The right panels of Figure 11 show the projected density of the $N$-body model at the end of the simulations. These profiles were fitted as a superposition of two model components, one intended to represent the galaxy and the other the NC. For both components we adopted the Sérsic law profile:

$$
\Sigma(R)=\Sigma_{0} \exp \left[-b\left(\frac{R}{R_{0}}\right)^{\frac{1}{n}}+b\right],
$$

with

$$
b=2 n-\frac{1}{3}+\frac{0.009876}{n} .
$$

For the end-product $N$-body model of run B, the best-fit parameters were $\Sigma_{0}=7.21 \times 10^{3} M_{\odot} \mathrm{pc}^{-2}, n=1.03$, and $R_{0}=31.9 \mathrm{pc}$ for the bulge and $\Sigma_{0}=1.03 \times 10^{4} M_{\odot} \mathrm{pc}^{-2}$, $n=1.88$, and $R_{0}=9.51 \mathrm{pc}$ for the NC. The best-fit parameters of the merger product of run $\mathrm{C}$ were $\Sigma_{0}=7.44 \times 10^{3} M_{\odot} \mathrm{pc}^{-2}$, $n=1.04$, and $R_{0}=31.6 \mathrm{pc}$ for the bulge and $\Sigma_{0}=$ $7.60 \times 10^{3} M_{\odot} \mathrm{pc}^{-2}, n=2.08$, and $R_{0}=11.3 \mathrm{pc}$ for the NC.

We note that the final density profile and structure of the $\mathrm{NC}$ depends on a variety of factors, which include the initial distribution of stars and BHs inside the clusters, the strength of the tidal field due to the galaxy and $\mathrm{MBH}$, and how the distribution of previously migrated stars and BHs evolves in response to their gravitational interaction with other background stars and with infalling clusters. For instance, the initial degree of internal evolution in the cluster models together with the adopted galactic $\mathrm{MBH}$ mass will determine how close to the galactic center the $\mathrm{BH}$ and stellar clusters will get before they completely dissociate; in turn this regulates the size of the region over which the stellar distribution flattens (i.e., the core size of the NC density profile), as well as the number of stellar BHs transported in the vicinity of the $\mathrm{MBH}$.

In Figure 12 the mass in BHs accumulated in the inner parsec, $M_{\mathrm{bh}}(<1 \mathrm{pc})$, is shown as a function of the NC mass, $M_{\mathrm{NC}}$. At any time the NC mass is given by the sum of the accumulated globular cluster masses. A good fit to the data for $M_{\mathrm{NC}}>$ $4 \times 10^{6} M_{\odot}$ is given by $M_{\mathrm{bh}}(<1 \mathrm{pc})=a \times\left(M_{\mathrm{NC}} / 4 \times 10^{6} M_{\odot}\right)^{b}$, with $a=3.33 \times 10^{4} M_{\odot}$ and $b=1.44$ for model $\mathrm{B}$ and $a=1.42 \times 10^{5} M_{\odot}$ and $b=0.819$ for model $\mathrm{C}$; these fitting functions are plotted in Figure 12 as solid curves.

The effect of the infalling clusters on the preexisting $\mathrm{NC}$ is illustrated in the left panel of Figure 13, which shows the density profile of stars in simulation B after 12 inspirals were completed and that were originally inside the 3rd, 6th, 9th, and 12th infalling cluster. The density profile of stars from the 12th merged cluster, consistently with the results of the high-resolution simulations of Figure 9, is characterized by a shallow density cusp out to roughly 1-2 pc and an outer envelope with density that falls off rapidly with radius. The dot-dashed curve in the figure illustrates the density profile of the same stars after the final NC model was run in isolation for a time equal to the time for three consecutive inspiral events to occur, $\sim 5 \times 10^{7}$ yr. The similarity between the two mass distributions 

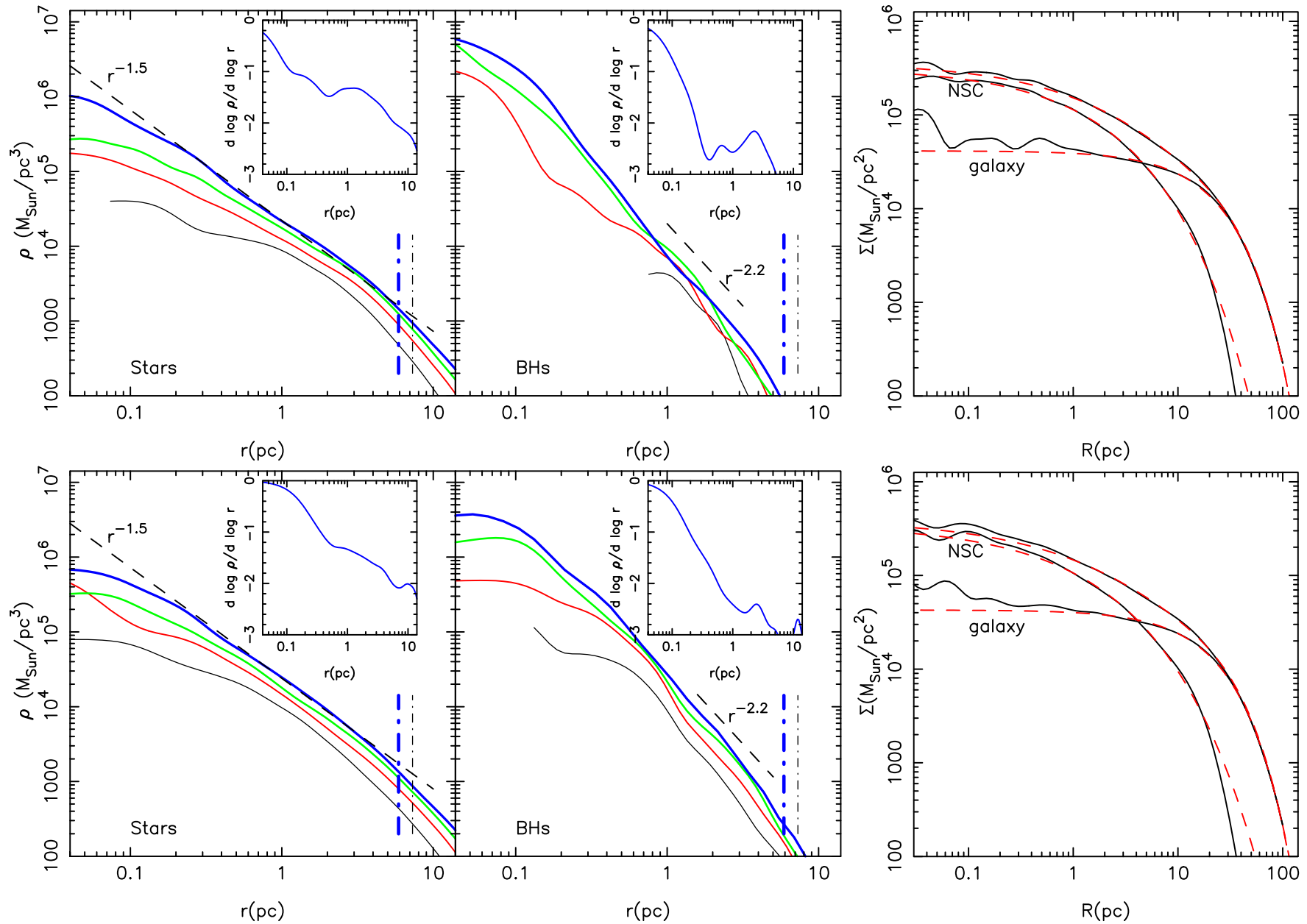

Figure 11. Density profile of the NC in simulations B (upper panel) and C (lower panel) during the globular cluster inspirals. The left panels give the density of the NC (i.e., only particles that were initially associated with the clusters are used) after 3, 6, 9, and 12 infall events (from bottom to top), while the middle panels show the corresponding density distribution of BHs. Dot-dashed curves give the radius containing a mass in stars twice the mass of the central MBH for the initial model (black dot-dashed curve) and for the simulation end-products (blue dot-dashed curve). The insert panels show the radial dependence of the density profile slope $d \log \rho / d \log r$ in the final NC models. The right panels display the projected density profile of the stars in the NC, in the galaxy (i.e., only particles that were initially associated with the galaxy), and the sum of them (upper curves). The dashed red curves are the best-fitting Sérsic profile models to these projected distributions.

(A color version of this figure is available in the online journal.)

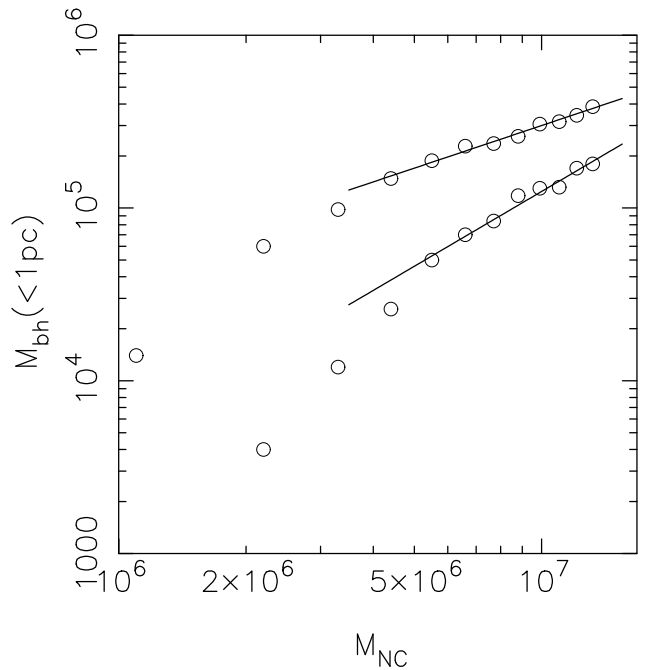

Figure 12. Total mass of BHs accumulated inside the inner parsec during the inspiral simulations B (lower points) and C (upper points), as a function of the mass of the NC (i.e., the sum of the decayed globular cluster masses). The solid curves give the best-fitting power-law profiles to the data for $M_{\mathrm{NC}}>4 \times 10^{6} M_{\odot}$. Masses are in units of solar masses.

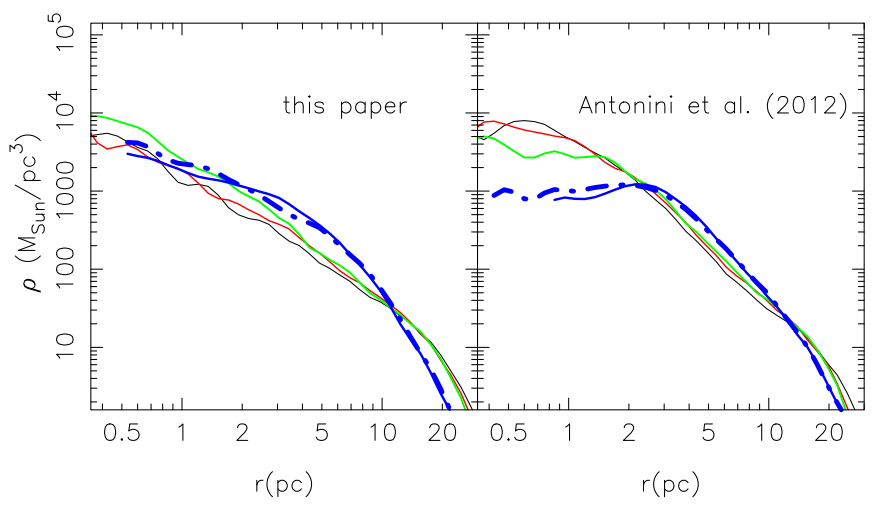

Figure 13. Left panel shows the density profile of stars in run B after 12 inspirals were completed and that were originally part of the 3rd, 6th, 9th, and 12 th infalling cluster. The right panel corresponds to the single-mass component simulations of Antonini et al. (2012). Blue dot-dashed curves show the density profile of stars coming from the 12th decayed cluster after the final NC model was run in isolation for a time corresponding to roughly the time that it takes our simulations for three consecutive inspiral events to complete.

(A color version of this figure is available in the online journal.) 
indicates that collisional two-body relaxation, due to random star-star and star-BH gravitational encounters, can be ignored during the inspiral simulations. The densities of stars coming from previously decayed globular clusters, however, appear very similar to each other and very different from the density of stars transported during the last inspiral, being lower and having a steeper radial dependence, $\mathrm{d} \log \rho / \mathrm{d} \log r \approx-1.5$, in the radial range $1 \mathrm{pc} \lesssim r \lesssim 10 \mathrm{pc}$.

As illustrated in the right panel of Figure 13, this evolution was also present in the one-component inspiral simulations of Antonini et al. (2012); the initial conditions of these simulations were essentially the same as those of simulation B with the difference that the clusters only had a single-mass particle group representing stars. There are two mechanisms that drive the evolution of the density profile toward their final form: (1) stars from earlier infalling clusters are stripped at smaller radii and dominate inner regions of the NC (Perets \& MastrobuonoBattisti 2014) and, as argued in the following section, (2) gravitational scattering of the previously accumulated stars and BHs by the infalling clusters.

\subsection{Accelerated Cusp Regrowth due to Scattering from Infalling Clusters}

Consider a massive cluster of mass $m_{\mathrm{cl}}$ that moves into a system of $N$ stars. The second-order diffusion coefficients that appear in the Fokker-Plank equation and that describe the evolution of the stellar distribution due to self-scattering $\left(\mathcal{D}_{E E, 11}\right)$ and scattering off of the cluster $\left(\mathcal{D}_{E E, 1 \mathrm{cl}}\right)$, scale with the mass of the perturber and the mass $(m)$ and density of field particles as

$$
\mathcal{D}_{E E, 11} \simeq m^{2} N ; \quad \mathcal{D}_{E E, 1 \mathrm{cl}} \simeq m_{\mathrm{cl}}^{2},
$$

where here we have approximated the cluster as a point mass perturber. From Equation (17) we see that if $m_{\mathrm{cl}} \gg \sqrt{m^{2} N}$, $\mathcal{D}_{E E, 1 \mathrm{cl}} \gg \mathcal{D}_{E E, 11}$, i.e., self-scattering is negligible compared with scattering off of the massive perturber; the first-order coefficients can also be ignored since they are smaller than $\mathcal{D}_{E E, 1 \mathrm{cl}}$ by factors of $m^{2} N / m_{\mathrm{cl}}^{2}$ and $m / m_{\mathrm{cl}}$. Thus, if

$$
m_{\mathrm{cl}}>\sqrt{m^{2} N}
$$

an inspiraling cluster will reduce the energy relaxation timescale compared with that due to stars alone by a factor $\sim m_{\mathrm{cl}}^{2} \ln \Lambda / m^{2} N \ln \Lambda^{\prime}$. In the latter expression, $\Lambda^{\prime}$ represents the Coulomb logarithm estimated by taking into account the large physical size of the cluster, which implies a lower effectiveness of close gravitational scattering with respect to star-star scattering (e.g., Merritt 2013). This latter quantity can be set to

$$
\ln \Lambda^{\prime} \approx \frac{1}{2} \ln \left[1+\frac{p_{\max }^{2}}{p_{0}^{2}}\right]
$$

with $p_{\max }$ roughly one-fourth times the linear extent of the test star's orbit. Setting this size to $5 \mathrm{pc}$, and $p_{0}$ to half of the size of the cluster, $\approx 1 \mathrm{pc}$, the typical relaxation time becomes

$$
\begin{aligned}
& T_{r}^{\mathrm{eff}}(r) \approx \frac{0.34 \sigma^{3}(r)}{G^{2} \ln \Lambda^{\prime} \rho} \frac{M_{\bullet}}{m_{\mathrm{cl}}^{2}}=2 \times 10^{7}\left(\frac{\sigma}{50 \mathrm{~km} \mathrm{~s}^{-1}}\right)^{3} \\
& \quad \times\left(\frac{1.6}{\ln \Lambda^{\prime}} \frac{10^{3} M_{\odot}}{\rho} \frac{M_{\bullet}}{4 \times 10^{6} M_{\odot}}\right)\left(\frac{5 \times 10^{5} M_{\odot}}{m_{\mathrm{cl}}}\right)^{2} \mathrm{yr},
\end{aligned}
$$

where in deriving this expression we have used the fact that $m N \approx M_{\bullet}$ near the sphere of influence of the $\mathrm{MBH}$.
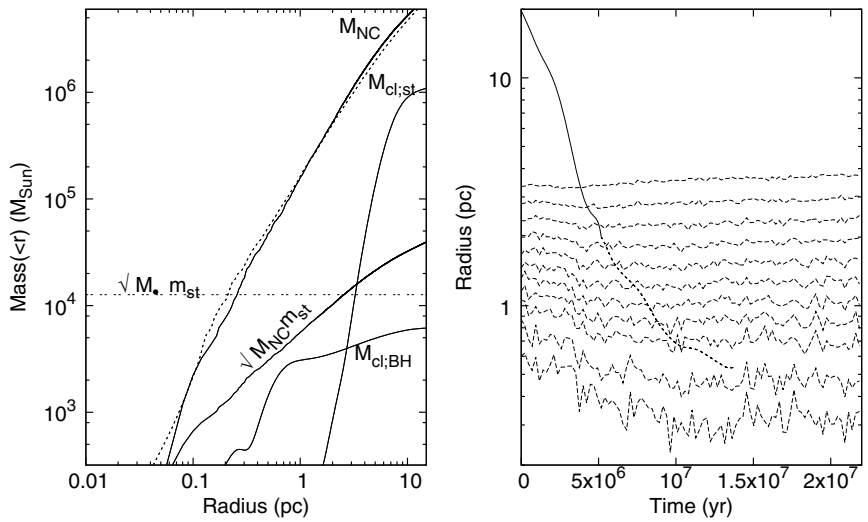

Figure 14. Left panel: upper curves display the cumulative mass distribution of stars $\left(M_{\mathrm{NC}}\right)$ in the $\mathrm{NC}$ at the beginning (solid curve) and at the end (dotted curve) of the 12th inspiral. The total mass in stars $\left(m_{\mathrm{cl}, \mathrm{st}}\right)$ and BHs $\left(m_{\mathrm{cl}, \mathrm{bh}}\right)$ as a function of the galactocentric radius of the 12 inspiraling clusters are displayed; time increases from right to left as the cluster orbit decays toward the center and loses mass. The value of $\sqrt{M_{\mathrm{NC}} m_{\mathrm{st}}}$ is also illustrated as a function of radius; this is roughly equal to $\sqrt{M_{\bullet} m_{\mathrm{st}}}$ (double-dotted curve) at the $\mathrm{MBH}$ radius of influence. When the total cluster mass is larger than $\sqrt{M_{\mathrm{NC}} m_{\mathrm{st}}}$, collisional relaxation in the $\mathrm{NC}$ is dominated by scattering of stars and $\mathrm{BHs}$ from the infalling cluster. In the right panel we display the Lagrangian radii of stars from the previously decayed (11th) cluster during the 12th inspiral (dashed curves) and the evolution of the orbital radius of the 12th cluster. The orbit of the cluster is shown using a continued curve when $m_{\mathrm{cl}}>\sqrt{M_{\mathrm{NC}} m_{\mathrm{st}}}$ and a dotted curve otherwise. The evolution of the Lagrangian radii clearly shows that the inspiral modifies the mass distribution of the preexisting $\mathrm{NC}$, as argued in the text.

For $M_{\bullet} \sim 10^{7} M_{\odot}$, the infall of even a cluster of $10^{4} M_{\odot}$ would largely affect the rate of collisional relaxation, provided that the cluster spends a time of the order of $T_{r}^{\text {eff }}$ in a region where the condition (18) is satisfied.

The left panel of Figure 14 shows the total mass of BHs and stars bound to the 12th infalling cluster in run B as a function of cluster orbital radius and compares these with $\sqrt{m^{2} N}$. The cluster spends roughly $5 \times 10^{6}$ yr before it reaches $\sim 2 \mathrm{pc}$ (right panel of Figure 14), after which its mass drops below $\sqrt{M_{\bullet} m}$ and self-scattering starts to be the dominant effect driving collisional relaxation. Since the time for the cluster to reach this radius is comparable to $T_{r}^{\text {eff }}$, the inspiral of clusters is expected to have an important impact on the density distribution of the preexisting $\mathrm{NC}$, in agreement with the results of our simulations. In the right panel of Figure 14 we show the evolution of the Lagrangian radii of the stars transported in the $\mathrm{NC}$ during the previous (11th) inspiral. During the first $10^{7} \mathrm{yr}$, the condition (18) is satisfied and the inspiral induces a rapid evolution of the NC mass distribution. Note that in a real galaxy, because of the smaller individual stellar mass, scattering from the perturber would dominate down to smaller radii than in our $N$-body model. However, in the region where the condition (18) is satisfied, the relaxation time in the simulations would be roughly the same as in the real system.

Scattering of stars by the massive perturber will cause an initial density core to fill up and the distribution function to evolve toward a constant value. The result is a sharp increase in the density profile of stars, $n \sim r^{-1.5}$, inside a radius approximately equal to the radius within which the condition Equation (18) is first met. After the perturber reaches a radius $r_{\text {crit }}$ containing a total mass in stars smaller than its mass, a large density core is rapidly carved out as a consequence of ejection of stars from the center. This is the evolution that, for example, characterizes the mass distribution of stars during the formation and evolution of MBH binaries in galactic nuclei (see Figures 13 
and 14 of Antonini \& Merritt 2012). In our simulations, however, the clusters disrupt before reaching $r_{\text {crit }}$ and before the second phase of cusp disruption can initiate. Thus, the net effect of infalling clusters on the preexisting $\mathrm{NC}$ distribution is that of inducing a short period of enhanced collisional relaxation, which causes the central density of stars and BHs to increase during the inspirals.

\subsection{Collisional evolution}

During and after its formation, an $\mathrm{NC}$ will evolve because of collisional star-star, star-BH, and $\mathrm{BH}-\mathrm{BH}$ interactions that will cause its density distribution to slowly morph into the steeply rising density profile, which describes the quasi-steadystate solution of stars and BHs near an $\mathrm{MBH}$. We study the evolution of the $\mathrm{NC}$ due to collisional relaxation by evolving the inspiral simulation end-products of simulation B for roughly half a relaxation time (or one Hubble time when scaling the $\mathrm{N}$-body model to the Milky Way). In order to efficiently evolve the system for such a long timescale, the $N$-body model was resampled to contain a smaller number of particles. Since we are interested in the model distribution at small radii, we kept the mass of the particles the same as in the original model, so that the resolution of the simulation in the region near the center was unchanged, and we included only particles with orbital periapsis less than $10 \mathrm{pc}$ and apoapsis less than $20 \mathrm{pc}$. In this way the total gravitational force acting on particles lying inside $\approx 10 \mathrm{pc}$ was approximately unchanged with respect to the original model. This sampling procedure is equivalent to truncating the mass distribution of the model smoothly at $r \gtrsim 10$ pc. $N$-body integrations over a few crossing times verified the (collisionless) quasi-equilibrium state of the truncated model.

Since the dynamical friction times for globular clusters at $20 \mathrm{pc}$ from the galactic center are much shorter than relaxation times, both the cluster and galaxy will not undergo a significant amount of collisional relaxation during the inspirals. In our $N$-body simulations B and $\mathrm{C}$, the inspiral time of the globulars to reach the center and disperse around the central $\mathrm{MBH}$ is about 100 times shorter than the relaxation time of the NC. Thus, during the inspiral simulations, relaxation due to star-star, star-BH, and $\mathrm{BH}-\mathrm{BH}$ interactions was ignored, while in the post-merger simulations presented here times were scaled to the relaxation time computed at the sphere of influence of the $\mathrm{MBH}$. Note that for the sake of simplicity, the evolution was broken into two successive stages: the infall of the clusters and then evolution, due to two-body encounters, of the stellar distribution around the MBH with infalls "turned off." In reality, subsequent inspirals would be separated by times of order a Gyr and significant two-body relaxation would occur between these events.

Figure 15 shows the evolution of the mass density in each species in the post-merger integrations. Initially, the density of BHs dominates over the density in stars at $r \lesssim 0.1 r_{\text {infl }}$. Thus, at radii smaller than these, the evolution of the $\mathrm{BHs}$ is mostly driven by $\mathrm{BH}-\mathrm{BH}$ self-scattering that causes their distribution to reach, in roughly $0.2 \times T_{r_{\text {inf }}}$, a quasi-steady-state form characterized by a steep density slope, $\gamma \approx 2.2$, at all radii. At radii larger than $\sim 0.1 r_{\text {infl }}$, the stars dominate the mass density and the $\mathrm{BH}$ population evolves because of dynamical friction against the lighter component. The general trend is for the central mass density of BHs to steadily increase with time.

While the BHs segregate to the center, the lighter particle mass density decreases within the radial range $0.1 \lesssim r \lesssim 10$ pc. The evolution of the stellar distribution toward lower densities



Figure 15. Evolution of the density profile of stars and $\mathrm{BHs}$ during the postmerger simulation of model $\mathrm{B}$ at $t=(0.09,0.18,0.27,0.36) \times T_{r_{\text {infl }}}$. Insert panels give the radial dependence of the density profile slope $d \log \rho / d \log r$ of the final model $\left(t=0.36 T_{r_{\text {infl }}}\right)$.

(A color version of this figure is available in the online journal.)

is a consequence of their gravitational interaction with the BHs: when the density in the BHs approaches locally the density in stars, heating of the light particles off the heavy particles dominates over star-star scattering, causing the density of the former to decrease. Scattering of stars off the BHs is also expected to promptly modify the star density profile at small radii, causing the formation of a "mini-cusp" at $r \lesssim 0.02 r_{\text {infl }}$ (Gualandris \& Merritt 2012). We find evidence of this phenomenon in our $N$-body models, which rapidly develop a $n \sim r^{-1.5}$ cusp at $r<0.03$ pc (see upper panel and corresponding insert panel of Figure 15). Outside these radii, however, even after about half a relaxation time the star distribution retains the shallow density profile at $r \lesssim 0.2 \mathrm{pc}$ that characterized the initial model. We conclude that even after a time of order the relaxation time, model B looks different from the dynamically relaxed models that are often assumed to describe the density distribution of stars and BHs near the center of galaxies. After $\approx 0.4 \times T_{r_{\text {infl }}}$, the BHs (stars) attain a central density cusp that is steeper (shallower) than in those models.

We note that the details of the final density distribution of our NC model depend on a number of factors that remain quite uncertain. For example, the final state of the $\mathrm{BH}$ population in the $\mathrm{NC}$ and the degree at which the $\mathrm{BHs}$ are segregated in 

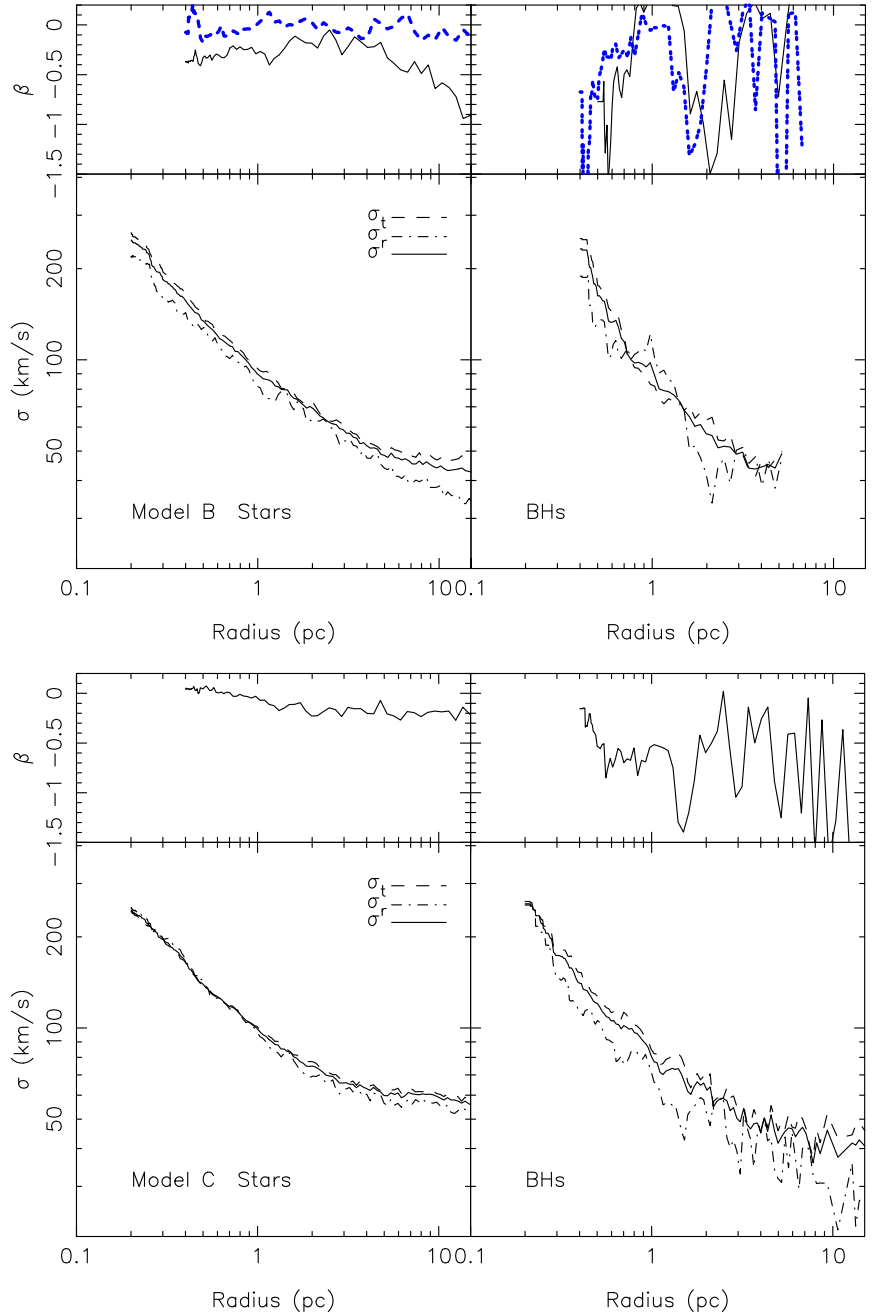

Figure 16. Radial profile of the anisotropy parameter $(\beta)$ and radial $\left(\sigma_{r}\right)$ and tangential $\left(\sigma_{t}\right)$ components of the one-dimensional velocity dispersion $(\sigma)$. Plots were obtained at the end of the 12th inspiral event by using only $N$-body particles that were originally inside the clusters. The blue dashed curves in the upper panels display the anisotropy profile of the $\mathrm{NC}$ in simulation B at the end of the post-merger phase.

(A color version of this figure is available in the online journal.)

the final model will depend on their initial number fraction and thus on the assumptions made for their initial distribution in the parent clusters.

\subsection{Kinematics and Morphology}

Understanding the kinematical structure and shape of galactic nuclei is important for placing constraints on their formation history and evolution. We quantified the velocity anisotropy of our models by using the parameter

$$
\beta=1-\frac{\sigma_{t}^{2}}{2 \sigma_{r}^{2}},
$$

where $\sigma_{r}$ and $\sigma_{t}$ are the radial and tangential velocity dispersions, respectively. Figure 16 shows the radial profile of $\beta, \sigma_{r}$, and $\sigma_{t}$ for the stellar and $\mathrm{BH}$ populations in simulations $\mathrm{B}$ and C. At the end of the inspiral simulations, model B is characterized by an approximately flat velocity anisotropy profile with $\beta \approx-0.5$, while in model $\mathrm{C} \beta$ slightly decreases from nearly 0 to -0.2 within $1 \mathrm{pc}$ and is approximately constant outside this radius. Thus, our models are tangentially anisotropic throughout
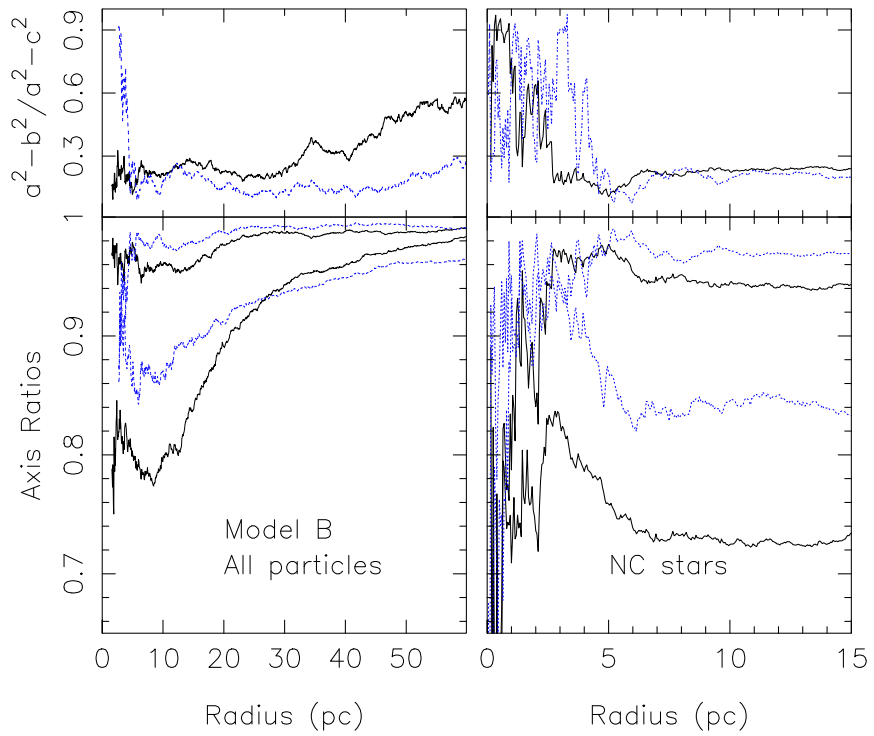

Figure 17. Triaxiality parameter (upper panel) and axis ratios (lower panel) as functions of radius, for model $\mathrm{B}$ at the end of the inspiral phase (black solid curves) and at the end of the post-merger collisional evolution (blue dotted curves). Left panels give the shape parameters obtained by including all particles (stars and BHs) in the evaluation of the symmetry axes; in the right panels only particles representing stars that were transported to the center by the infalling stellar clusters were used.

(A color version of this figure is available in the online journal.)

$100 \mathrm{pc}$, both in the stellar and BH components. The upper panels of Figure 16 also display the radial profile of the anisotropy parameter of model B at the end of the post-merger phase, i.e., after the system was run in isolation for a time of order the relaxation time. Evidently, two-body relaxation causes $\beta$ to increase and the NC to evolve toward spherical symmetry in velocity space.

We measured the model shape in our simulations from the moment-of-inertia tensor (e.g., Katz 1991; Poon \& Merritt 2004; Antonini et al. 2009). The symmetry axes are calculated as

$\tau_{1}=\sqrt{I_{11} / I_{\max }}, \quad \tau_{2}=\sqrt{I_{22} / I_{\max }}, \quad \tau_{3}=\sqrt{I_{33} / I_{\max }}$,

where $I_{i i}$ is the principal moments of the inertia tensor and $I_{\text {max }}=\max \left\{I_{11}, I_{22}, I_{33}\right\}$; particles are then enclosed within the ellipsoid $x^{2} / \tau_{1}{ }^{2}+y^{2} / \tau_{2}{ }^{2}+z^{2} / \tau_{3}{ }^{2}=r^{2}$. These previous steps were iterated until the values of the axial ratios had a percentage change of less than $10^{-2}$. We also define a triaxiality parameter: $T \equiv\left(a^{2}-b^{2}\right) /\left(a^{2}-c^{2}\right)$. The value $T=0.5$ corresponds to the 'maximally triaxiality'case, while oblate and prolate shapes correspond to $T=0$ and 1 , respectively.

Figure 17 displays the radial profile of the axis ratios and triaxiality parameter of the model in simulation B at the end of the inspiral simulation (black solid curves) and at the end of the post-infall evolution (blue dotted curves). We also evaluated the shape of the NC component by only including the stars that were transported to the center from the infalling stellar clusters (right panels); the NC component appears strongly triaxiallike within $5 \mathrm{pc}$ and mildly triaxial (quasi-oblate) outside this radius. The formed NCs in simulations $\mathrm{C}$ and $\mathrm{B}$ shared a similar morphological structure, so we only displayed results for model B. The model morphology within $\sim 30 \mathrm{pc}$ is initially mildly triaxial and evolves because of gravitational encounters toward a more quasi-spherical shape. It is important that at the end of the post-merger phase the model still exhibits a significant degree of triaxiality, $0.1 \lesssim T \lesssim 0.3$. In fact, such a level of asymmetry might be large enough to significantly increase the number 
of tidal captures of stars and stellar binaries when compared with the same rate obtained in collisionally resupplied loss cone theories where spherical geometry is often assumed both in configuration and velocity space (Merritt \& Vasiliev 2011).

\section{DISCUSSION}

\subsection{Comparing with the Properties of the Galactic Nuclear Cluster}

Star counts using adaptive optics spectroscopy and mediumband imaging have shown that the red giants at the Galactic center, the only old stars that can be resolved in these regions, have a flat projected surface density profile close to Sgr A* (Buchholz et al. 2009; Do et al. 2009). The core in the red giant population extends out to approximately $0.3-0.5 \mathrm{pc}$ from the center. However, because of the effect of projection, it is difficult to constrain the core size and three-dimensional spatial density profile, which could be slowly rising or declining toward the center.

It is possible that a cusp in the lower mass stars is present and that the observed core is the result of a luminosity function that changes within $0.5 \mathrm{pc}$. This could be due to physical collisions before or during the giant phase (Bailey \& Davies 1999; Dale et al. 2009) or tidal interactions between stars and the central MBH (Davies \& King 2005)_mass removal can make the luminosity that a star would otherwise reach at the tip of the red-giant phase considerably fainter and prevent these objects from evolving to become observable. While these models are possible, they seem to not fully explain the observations (Dale et al. 2009). Thus, it is important to consider the possibility that the red giants are indeed representative of the unresolved low-mass main-sequence stars and that the density core is a consequence of the $\mathrm{NC}$ formation history combined with a long nuclear relaxation timescale (Section 2). On this basis we can directly compare the predictions of theoretical models with the kinematics and mass distribution inferred from observations of the giant stars at the GC and draw conclusions about possible formation mechanisms for the Milky Way NC.

\subsubsection{Mass Distribution}

Figure 11 shows that the merger of about 10 clusters results in a compact nucleus with a stellar density profile that declines as $n \sim r^{-1.5}$ outside $\sim 0.5 \mathrm{pc}$ and flattens to $n \sim r^{-1}$ within this radius. More precisely, we consider two definitions of the core radius: (1) the projected radius, $r_{c}$, at which the surface density falls to one-half its central value and (2) the break radius, $r_{b}$, at which the density profile transits from the inner law $\left(n \sim r^{-1}\right)$ to the outer density law $\left(n \sim r^{-1.5}\right)$. We find $r_{c}=0.51 \mathrm{pc}$ and $r_{b}=0.48 \mathrm{pc}$ for model B and $r_{c}=0.61 \mathrm{pc}$ and $r_{b}=0.59 \mathrm{pc}$ for model $\mathrm{C}$. Collisional relaxation occurring during the post-merger simulation of model B reduces the size of the core with time, while inner and outer density slopes remain roughly unchanged. At the end of the simulation, after $\sim 0.3 T_{\mathrm{r}_{\mathrm{infl}}}$, we find $r_{c}=0.32 \mathrm{pc}$ and $r_{b}=0.18 \mathrm{pc}$. The size of the region where the density transits to a shallow profile in our models appears to be comparable to the extent of the core inferred from number counts of the red giant stars at the GC. The exact extent of the core region (and how far our models are from their steady state) is determined by a number of factors that depend on the assumptions made in the $N$-body initial conditions. For example, the size of the density core could be made larger or smaller depending on the initial degree of cluster evolution and the number fraction of BHs. However, we note that the presence of a core in the final density distribution seems to be a quite robust outcome of a merger model for NCs. For example, the single-mass component simulations of Antonini et al. (2012) also produced a final NC model with a core of size $\sim 1 \mathrm{pc}$, somewhat similar to what we find in this paper. The absence of a Bahcall-Wolf cusp is naturally explained in these models, without the need for fine-tuning or unrealistic initial conditions.

Our simulations result in a final density profile having nearly the same power-law index beyond $0.5 \mathrm{pc}$ as observed $\left(\Sigma(R) R^{-1}\right.$; Haller et al. 1996). The slope index in the inner $\sim 0.5 \mathrm{pc}$ of our model, $\gamma \sim 1$, is also consistent with what is obtained from the surface brightness distribution of stellar light within the inner 1" of Sgr A* (Yusef-Zadeh et al. 2012), but appears only marginally consistent with what is inferred from number counts of the red giant stars. Slope indexes in the range $-3 \lesssim \gamma \lesssim 0.8$ are consistent with what is derived from observations of the giants, although negative or nearly zero values corresponding to centrally decreasing or flat densities, respectively, are preferred (Merritt 2010; Do et al. 2013).

\subsubsection{Kinematics}

The radial profile of the velocity anisotropy of the NC could potentially provide a useful constraint on its formation. Kinematic modeling of proper motion data derived from the dominant old population of giants reveals a nearly spherical central cluster exhibiting slow, approximately solid body rotation of amplitude $1.4 \mathrm{~km} \mathrm{~s}^{-1} \operatorname{arcsec}^{-1}$ (Trippe et al. 2008; Schödel et al. 2009). Kinematically, the central cluster appears isotropic, with a mildly radial anisotropy at $r \lesssim 0.1 \mathrm{pc}$ and slightly tangentially anisotropic for $0.1 \mathrm{pc} \lesssim r \lesssim 1 \mathrm{pc}$. In the radial range $1^{\prime \prime}-10^{\prime \prime}$, the late-type stars are observed to have a mean projected anisotropy of $\left\langle 1-\sigma_{T}^{2} / \sigma_{R}^{2}\right\rangle=-0.12_{-1.05}^{+0.098}$ (Schödel et al. 2009). Do et al. (2013) found $\beta=0.01_{-0.34}^{+0.35}$ within $0.5 \mathrm{pc}$.

Our models are characterized a generally flat anisotropy profile with $\beta \approx-0.5$ at the end of the inspiral simulations and $\beta \approx 0$ at the end of the post-merger evolution. Although such values are consistent with observations, we believe that future and better kinematic data that extend outside the inner parsec will be necessary in order to provide better constraints on this scenario.

We note that because of our assumption of no preferential direction of inspiral, the merger remnants in our simulations showed no significant net rotation. Recent observations of the $\mathrm{NC}$ in our Galaxy suggest instead a significant rotation on parsec scale (Schödel et al. 2014); this might be reconciled with a cluster merger origin for the Galactic NC if, for instance, the clusters were originally dragged down into the Galactic disk plane (where they experience a greater dynamical friction force) and transported into the central region of the Galaxy where they then accumulated to form a dense nucleus that will then appear to rotate in the same sense of the Galaxy.

\subsubsection{Kinematically Cold Sub-structures}

Feldmeier et al. (2014) found indications for a sub-structure in the Galactic NC that is rotating approximately perpendicular to the Galactic rotation with $\sim 30 \mathrm{~km} \mathrm{~s}^{-1}$ at a distance of $\sim 20^{\prime \prime}$ or $0.8 \mathrm{pc}$ from $\mathrm{Sgr} \mathrm{A}^{*}$. In addition they found an offset of the rotation axis from the photometric minor axis and argue that this hints to infalling clusters.

We look for kinematic sub-structures in our models by using the Rayleigh (dipole; Rayleigh 1919) statistics $R^{\prime}$ defined as the length of the resultant of the unit vectors $l_{i}, i=1, \ldots, N$, where 


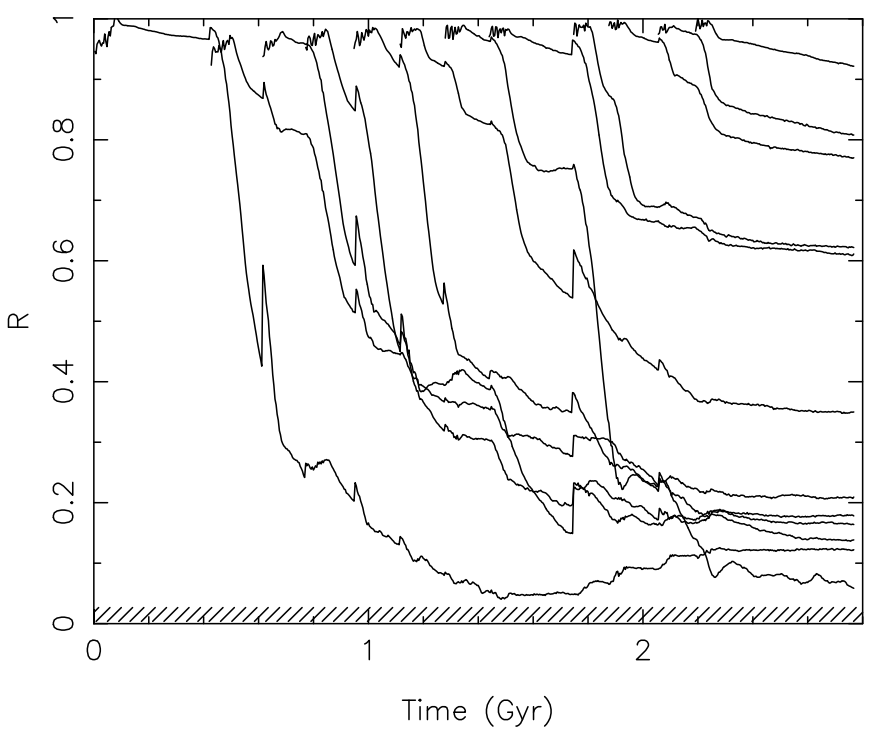

Figure 18. Evolution of the Rayleigh parameter $R=R^{\prime} / N$ that measures the degree of randomness of the orbital orientation of stars transported to the center by each of the 12 infalling clusters of model $\mathrm{B}$. The hatched region shows the $90 \%$ confidence bands expected for a fully random distribution of orbital orientations of 5715 stars. At the end of the 12th inspiral event, significantly cold kinematic sub-structures are present in the NC model.

$l_{i}$ is perpendicular to the orbital plane of the $i$ th particle and $N$ is the number of particles (a total of 5715 per cluster). For each merged cluster, we computed $R^{\prime}$ over the entire course of the inspiral simulation. For a fully isotropic distribution, we expect $R^{\prime} \sim \sqrt{N}$, while $R^{\prime} \sim N$ if the orbits are correlated

As an example, Figure 18 gives $R\left(=R^{\prime} / N\right)$ as a function of time (scaled to the relaxation time of a Milky Way-like nucleus). Initially, after a cluster reaches the center the orbits are strongly correlated and, as expected, $R \sim 1$, i.e., the stars from the infalling clusters distribute into a thin disk configuration initially. Because of two-body relaxation, and because of the perturbing effect of the later infalling clusters, $R$ decreases with time and approaches a value more consistent with isotropy. Figure 18 shows that all clusters maintain some degree of anisotropy during the entire course of the simulation. However, the orbits of the stars from the first seven clusters are almost completely isotropic by the end of the simulation. The orbits of stars from the last four/five infalling clusters are still largely correlated after $\sim 3$ Gyr of evolution. A linear fit to the data $(R$ versus $t$ ) for the last decayed cluster gives $R(t)=$ $-0.11 \times t / \mathrm{Gyr}+0.96$, so that it would take about $\sim 10 \mathrm{Gyr}$ for the stars to achieve a nearly isotropic distribution. These results are consistent with those of Mastrobuono-Battisti \& Perets (2013), who found that it takes a time of order the relaxation time of the nucleus to fully randomize an initially cold disk.

\subsection{Extreme Mass Ratio Inspirals}

The inspiral of compact remnants into an MBH represents one of the most promising sources of gravitational wave radiation detectable by space-based laser interferometers (Amaro-Seoane et al. 2012). Event rates for such extreme mass ratio inspirals are generally estimated under the assumption that the BHs had enough time to segregate and form a steep central cusp, $n \sim r^{-2}$, near the $\mathrm{MBH}$. Such dynamical models predict inspiral rates per galaxies of $\sim 250 \mathrm{Gyr}^{-1}$ (Hopman \& Alexander 2006). Models that include an initial parsec-scale core can result in much lower central $\mathrm{BH}$ densities than in the steady-state models and imply rates as low as $1-10 \mathrm{Gyr}^{-1}$ (Merritt 2010). EMRI event rates could be also severely suppressed by the Schwarzschild barrier, which limits the ability of stars to diffuse to high eccentricities onto inspiral orbits (Merritt et al. 2011).

It must be stressed that it is difficult to draw any conclusion about EMRI event rates from the models discussed in the literature because of the significant uncertainties in the underlining assumptions. Our simulations cast further doubts on results obtained from idealized time-dependent models, which rely on the assumption that BHs and stars have initially the same spatial distribution. For example, on the basis of the models discussed in Section 2 and in Merritt (2010) and Antonini \& Merritt (2012), the presence of a core in the old population of stars at the GC would imply very low central densities of BHs and EMRI event rates. However, the initial conditions adopted in the simulation of Section 2 were quite artificial and not motivated by any specific physical model. We have shown that in a merger model for the formation of NCs, the resulting distribution of stellar remnants partially reflects their distribution in their parent clusters just before they reach the center of the galaxy. Thus, different, possibly more realistic, initial conditions would produce rather different central BH densities; in these models EMRI rates could be as large as (or higher than) those obtained in the steady-state models even in the presence of a core in the stellar distribution.

In order to determine the number of BHs in a Milky Way-like nucleus predicted by the inspiral simulations, we used the scaling relation given by Equation (13). We are interested in the number of compact remnants inside $\approx 0.01 \mathrm{pc}$, as these are the only BHs that can generate EMRIs. Since at these radii the number of $\mathrm{BH}$ particles in the $N$-body model is small, we carried out regression fits of $\log N_{\text {bh }}$ to $\log r$ at larger radii and extrapolate inward in order to get $N_{\mathrm{bh}}$ at the radii of interest. Following Gualandris \& Merritt (2012), we performed these fits in the radial interval $\left(r_{1}, r_{2}\right)$ such that $N_{\mathrm{bh}}\left(<r_{1}\right)=5$ and $N_{\text {bh }}\left(<r_{2}\right)=25$, and assume a density profile of constant powerlaw index. In Figure 19 we show the (scaled) cumulative number of BHs during the post-merger simulations of Section 5.4. We find $N_{\mathrm{bh}}(<0.01 \mathrm{pc}) \approx 200$ at $t=0.1 \times T_{r_{\text {infl }}}$ and $N_{\text {bh }}(<0.01 \mathrm{pc}) \approx 400$ at $t=0.3 \times T_{r_{\text {infl }}}$. We can directly compare the numbers so obtained with estimates from quasisteady-state Fokker-Plank models of the GC. In their models, Hopman \& Alexander (2006) assumed $f=0.01$ and found $N_{\text {bh }}(r<0.01 \mathrm{pc})=150$; Freitag et al. (2006) found similar values. This is similar to the number of BHs in our model at the end of the inspiral simulations but somewhat smaller than the predicted number of $\mathrm{BHs}$ at the end of the post-merger simulations.

In conclusion, a core in the density distribution of stars does not necessarily imply a low density of stellar remnants in the GC. In a merger model for NCs, BHs initially have a smaller core as a relic of their premerger mass segregation, which was not fully erased by the tidal disruption process of the cluster. After a small fraction of the relaxation time $\left(\sim 0.1 T_{\mathrm{r}_{\text {inf }}}\right)$, the $\mathrm{BHs}$ dynamically relax because of $\mathrm{BH}-\mathrm{BH}$ interactions and attain a steep central density cusp, while the core in the stellar distribution persists. After this time, the number of BHs inside $0.01 \mathrm{pc}$, the radii relevant for EMRIs, is $N_{\mathrm{bh}}(<0.01 \mathrm{pc}) \sim 100$, a value comparable to that inferred in steady-state models.

\subsection{Constraining the Number of Dark Remnants at the GC using the $S$-star Orbits}

Observations of the Galactic center have revealed the existence of $\sim 20$ young (B-type) stars orbiting within $\sim 0.05 \mathrm{pc}$ of 


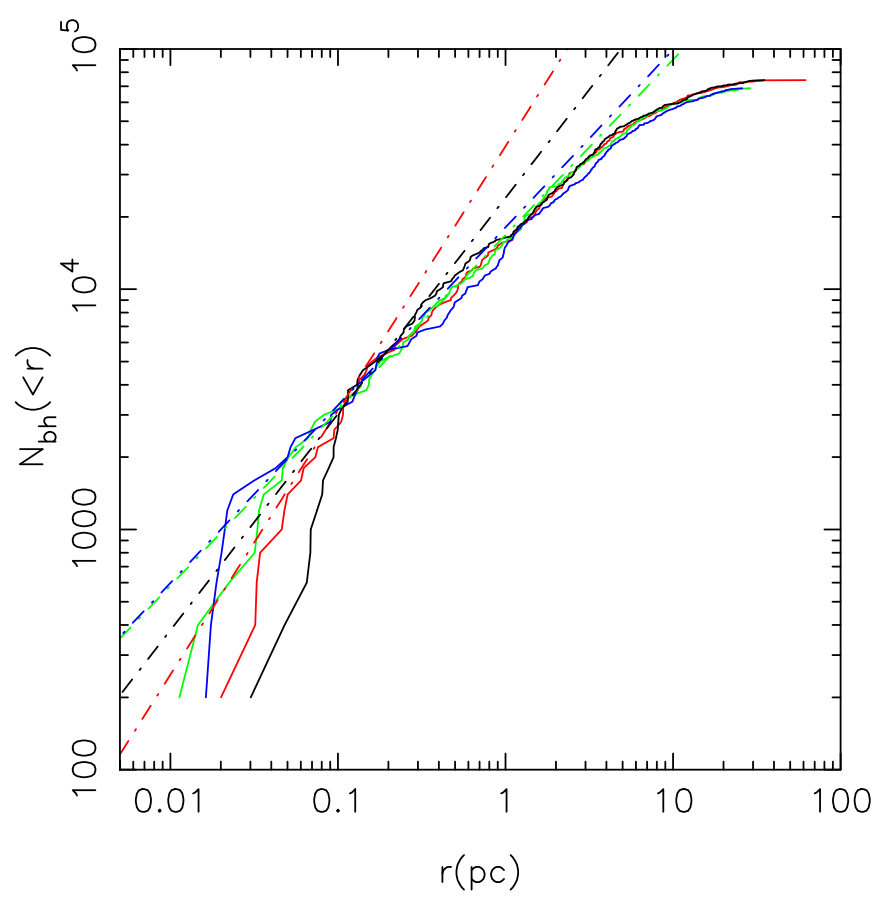

Figure 19. Cumulative number of BHs predicted for a Milky Way nucleus as a function of radius and at the different time intervals, $t=$ $(0.09,0.18,0.27,0.36) \times T_{r_{\text {infl }}}$, for the post-merger simulation discussed in Section 5.4. Dot-dashed curves were obtained by carrying out regression fits of $\log N_{\mathrm{bh}}$ to $\log r$ in the radial range $\left(r_{1}, r_{2}\right)$, with $N_{\mathrm{bh}}\left(<r_{1}\right)=5$ and $N_{\text {bh }}\left(<r_{2}\right)=25$ (see text for details).

(A color version of this figure is available in the online journal.)

the central black hole, the S stars (Ghez et al. 2008; Gillessen 2009). The $S$ stars are thought not to have formed in situ where the strong tidal field of the $\mathrm{MBH}$ would prevent star formation (Morris 1993). Mechanisms that invoke the formation of the $\mathrm{S}$ stars farther from the $\mathrm{MBH}$ and subsequent rapid migration to their current location are often invoked in order to explain their existence. The current paradigm is that the $\mathrm{S}$ stars result from the capture of individual stars by the tidal disruption of binaries following a close encounter to the central black hole (Hills 1988). This formation model can successfully account for the number of observed S stars (Perets et al. 2007), but it results in an orbital distribution that is largely biased toward high eccentricities, and therefore it is inconsistent with the quasi-thermal eccentricity distribution of the S star orbits (e.g., Antonini et al. 2010). Post-migration dynamical evolution due to gravitational perturbations from a field population of BHs has been invoked in order to bring the predicted orbital distributions more in line with observations (Perets et al. 2009; Madigan et al. 2011; Hamers et al. 2014). It has been recently shown that such a scenario could indeed lead to an orbital distribution that reproduces the random and eccentric character of the observed orbits (Antonini \& Merritt 2013).

The timescale over which the S-star orbits evolve and approach the observed distribution depends on the number of objects, i.e., BHs, in the stellar cusp, in which the larger the number of BHs is, the shorter the timescale is (Antonini \& Merritt 2013). Accordingly, we determine a lower limit to the number of BHs in the GC by following the dynamical evolution of the $\mathrm{S}$ stars for different values of $N_{\text {bh }}$ and by requiring the orbits to approach the observed distribution in $\sim 100 \mathrm{Myr}$, the main-sequence life time of a B-type star.
We ignore two-body relaxation since the timescale of interest ( $100 \mathrm{Myr}$ ) is short compared with two-body (non-resonant) relaxation times near the center of the Milky Way (Merritt 2010). Following Antonini \& Merritt (2013) we assume that the semi-major axis distribution, $N(a)$, is known, and it is given by the observed values of $a$. The initial orbital eccentricities were assigned randomly in the range $0.93 \leqslant e \leqslant 0.99$. The orbits were initialized at random times between 0 and $100 \mathrm{Myr}$ and followed to a final time of 100 Myr. This setup approximately reproduces the initial conditions expected in a binary disruption scenario for the formation of the S-stars (Zhang et al. 2013). For each S star (i.e., for each value of a), 100 integrations were carried out using the same Hamiltonian model adopted in Merritt et al. (2011) and Antonini \& Merritt (2013). Briefly, the Hamiltonian model accounts for the torques due to finite- $N$ asymmetries in the field-star distribution (resonant relaxation) and 1-PN terms that result in the (Schwarzschild) precession of the argument of periastron. The direction of the torquing field was changed smoothly with time and was randomized in a time of order the precession time of the field population as described in Section VB of Merritt et al. (2011).

We consider a field population of $10 M_{\odot}$ BHs, which we distributed according to a power-law density profile $\rho \sim r^{-2}$. The number of BHs at radii less than $r$ is

$$
N_{\mathrm{bh}}(<r)=N_{0.1}(r / 0.1 \mathrm{pc})
$$

where $N_{0.1}$ is the number of $\mathrm{BHs}$ within a radius of $0.1 \mathrm{pc}$; we take $N_{0.1}=(100,250,500,700,1000)$, and for each of these values we compared the results of our simulations with observations by performing a two-sample Kolmogorov-Smirnov (K-S) test on the eccentricity distributions.

In any of our cusp models, most orbits have orbital eccentricities that lie initially inside (i.e., at higher eccentricity than) the Schwarzschild barrier-the locus of points in the $(E, L)$ plane where resonant relaxation is suppressed by fast relativistic precession of a test particle orbit (Merritt et al. 2011). As shown in Antonini \& Merritt (2013), the Schwarzschild barrier behaves as a one-way permeable membrane, which is penetrable by orbits that approach it from higher eccentricities, while it is a hard barrier for orbits that approach it from above. It can be shown that the time to diffuse above the Schwarzschild barrier, toward higher angular momenta, scales approximately with the number of perturbers as $T_{d} \sim N_{\mathrm{bh}}^{-3 / 2}(<a)$ or as $\sim N_{\mathrm{bh}}^{-2}(<a)$ if the choice for the coherence time is the "vector" resonant relaxation time or the mass precession time, respectively-i.e., the test particles diffuse faster toward larger angular momenta, above the Schwarzschild barrier, the larger the number of background perturbers is (Figure 20).

Figure 21 shows the cumulative eccentricity distributions at $5 \times 10^{7} \mathrm{yr}$ and at $10^{8} \mathrm{yr}$ of evolution for various values of $N_{0.1}$. As the number of BHs in the cusp increases, the diffusion timescale decreases, allowing the distribution to more rapidly approach a quasi-thermal form. The insert panels give the $p$-value of the K-S tests as a function of $N_{0.1}$. Only for $N_{0.1} \gtrsim 1000$ after $\sim 100 \mathrm{Myr}$ does the eccentricity distribution appear to have approached a form that is consistent with observations ( $p$-value $\gtrsim 0.1$ ). These results imply that in order to explain the quasi-thermal character of the S-star orbital eccentricities, the number of BHs within $\sim 0.1 \mathrm{pc}$ of $\operatorname{Sgr} \mathrm{A}^{*}$ has to be $\gtrsim 1000$. Interestingly, this number is in agreement with the predictions of our globular cluster merger model (Figure 15), but it appears to be much larger than the number of BHs implied by the dynamical models discussed 


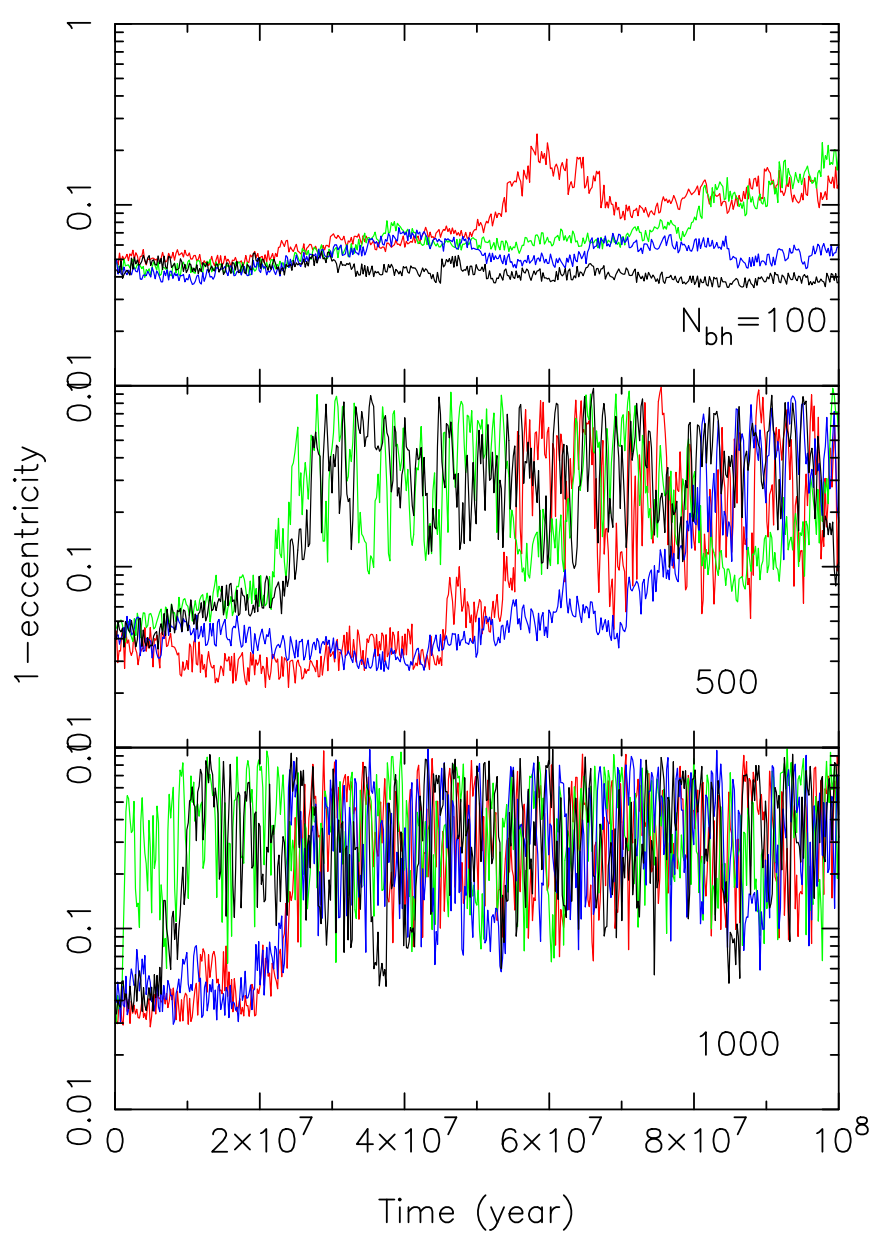

Figure 20. Eccentricity evolution of test particles taking different numbers of $10 M_{\odot}$ perturbers: $N_{0.1}=100,500,1000$. The initial values of the orbital elements were $a=10 \mathrm{mpc}, e=0.95, \Omega=0, \omega=-\pi / 2$, and $i=0.35 \pi$. The diffusion timescale to evolve to higher angular momenta, where resonant relaxation becomes more efficient in randomizing orbital eccentricities, decreases the larger the number of $\mathrm{BHs}$ in the cusp is.

(A color version of this figure is available in the online journal.)

in Section 2, which for $f_{\mathrm{bh}}=10^{-3}$ give $N_{0.1} \approx 100$ after $10 \mathrm{Gyr}$ of evolution.

Interestingly, a globular cluster merger model for the Milky Way NC can potentially reconcile models that require high central densities of BHs in order to explain the orbits of the $\mathrm{S}$ stars with the "missing cusp" problem of the giant star population.

\section{SUMMARY}

Understanding the distribution of stellar BHs at the center of the Galaxy is fundamental for a variety of astrophysical problems. These problems include the randomization of the $\mathrm{S}$-star orbits, the warping of the young stellar disk, and the inspiral of BHs into MBHs - an important class of gravitational wave sources for the future space-based interferometer antenna eLISA (Amaro-Seoane et al. 2012). The efficiency of these processes is very sensitive to the number of BHs and to their density distribution near the GC. In this paper we have used $\mathrm{N}$-body simulations to follow the evolution of BHs in models that have not yet reached a collisional steady state. Following the evolution of the BHs for timescales of order the age of the Galaxy, we made predictions about their density distribution under the two assumptions that (1) they follow the same phase-
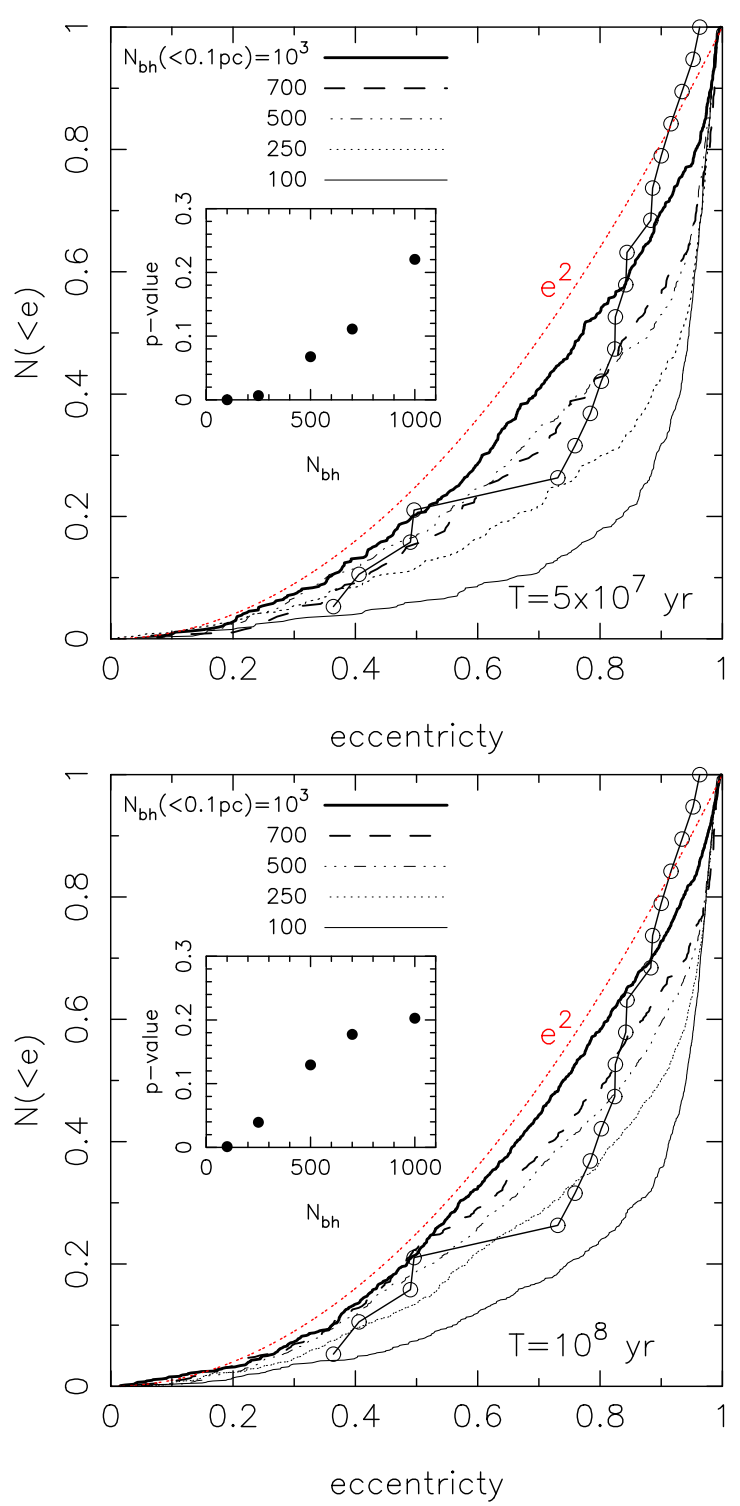

Figure 21. Cumulative eccentricity distributions at $5 \times 10^{7} \mathrm{yr}$ and at $10^{8} \mathrm{yr}$ of evolution for various values of the number of $10 M_{\odot} \mathrm{BH}$ perturbers inside $0.1 \mathrm{pc}$, $N_{0.1}$. The insert panels give the $p$-value of the K-S tests as a function of $N_{0.1}$. Only when $N_{0.1} \gtrsim 1000$ does the eccentricity distribution appear to approach a form that is consistent with observations ( $p$-value $\gtrsim 0.1$ ) after $\sim 100 \mathrm{Myr}$ of evolution (the S-star lifespan).

(A color version of this figure is available in the online journal.)

space distributions initially as the stars; and (2) they are initially brought into the Galactic center by dynamically evolved massive clusters. Our main results are summarized below.

1. We evolved models that have a parsec-scale density core and in which the BHs have the same phase-space distribution initially as the stars. We found that the time required for the growth of a relaxed, mass-segregated stellar cusp is shorter in models that contain a population of heavy remnants (e.g., Figure 2), and it is sensitive to their initial number relative to the stars.

2. Over the age of the Galaxy, and for a standard IMF, scattering off the BHs has little influence on the evolution of the lighter species. The time required for the regrowth of a mass-segregated stellar cusp can be longer than the Hubble time for galaxies similar to the Milky Way (e.g., Figure 3). 
3. In low and intermediate luminosity galaxies, globular clusters can decay to the center of the galaxy through dynamical friction and form a compact NC (Figure 5). Such clusters may harbor an inner core cluster of BHs that formed and mass-segregated to the center during the cluster evolution. For sufficiently massive clusters, the BH population is likely to be retained in the cluster center and then transported to the inner regions of the galaxy. Thus, massive clusters can represent an efficient source of BHs in the central regions of galaxies.

4. We used direct $N$-body simulations to follow the inspiral and merger of globular clusters in the GC. These clusters contained two stellar populations, representing starts and BHs. Both standard and top-heavy mass functions were considered. The BHs were initially segregated to the cluster center. After about 10 inspiral events, the formed NC develops a density profile that falls off as $\sim r^{-1.5}$ and a shallower core-like profile, $\gamma \lesssim 1$, inside a radius $\sim 0.5 \mathrm{pc}$. These properties are similar to those observed in the Milky Way NC. We find that the initial mass-segregation is not completely erased as the clusters are disrupted by the MBH tidal field (Figure 10). As a consequence of this, in the merger end-product the BHs dominate the total mass density within a radius of approximately $0.3 \mathrm{pc}$ (Figure 11).

5. By continuing the evolution of the model after the final inspiral event, we find that the $\mathrm{BH}$ population rapidly relaxes and attains a steep central density cusp, $r^{-2.2}$. By half a relaxation time, or roughly $10 \mathrm{Gyr}$ (when scaled to the Milky Way), the core that was formed in the stellar distribution shrinks to $0.1-0.2 \mathrm{pc}$ (Figure 15). While the density profile slope outside these radii remained nearly unchanged, the densities decreased as a consequence of heating of the stars by the BHs. Gravitational encounters also caused the NC to evolve toward spherical symmetry in configuration and velocity space.

6. We studied the orbital evolution of the $\mathrm{S}$ stars under the assumption that they were deposited to the GC through the disruption of binary stars by the MBH. Our analysis included the joint effects of Newtonian and relativistic perturbations to the motion, including the torques due to finite- $N$ asymmetries in the field-star distribution (resonant relaxation). We evolved the S-star orbits for a time of order $10^{8} \mathrm{yr}$, adopting models for the GC characterized by different number densities of BHs. We find that in order for the $\mathrm{S}$ stars to achieve a nearly thermal distribution of eccentricities during their lifetime, the GC should contain $\gtrsim 1000 \mathrm{BHs}$ inside $0.1 \mathrm{pc}$ (Figure 21). We argue that this lower limit for the number of BHs at the GC is consistent with a dissipationless formation model for the origin of the Milky Way NC.

I am grateful to R. Capuzzo-Dolcetta, D. Merritt, and A. Mstrobuono-Battisti for numerous discussions about the ideas presented in this paper. I thank D. Merritt and H. Perets for useful comments that helped to improve an earlier version of this manuscript. This research was partially supported by the National Aeronautics and Space Administration under grant No. NNX13AG92G. Computations were performed on Sunnyvale computing cluster at CITA and also on the ARC supercomputer at the SciNet HPC Consortium. SciNet is funded by: the Canada Foundation for Innovation under the auspices of Compute Canada, the Government of Ontario, Ontario Research Fund Research Excellence, and the University of Toronto.

\section{REFERENCES}

Alexander, T. 2005, PhR, 419, 65

Alexander, T., \& Hopman, C. 2009, ApJ, 697, 1861

Amaro-Seoane, P., Aondia, S., Babak, S., et al. 2012, CQGra, 29, 124016

Antonini, F. 2013, ApJ, 763, 62

Antonini, F., Capuzzo-Dolcetta, R., Mastrobuono-Battisti, A., \& Merritt, D. 2012, ApJ, 750, 111

Antonini, F., Capuzzo-Dolcetta, R., \& Merritt, D. 2009, MNRAS, 399, 671

Antonini, F., Faber, J., Gualandris, A., \& Merritt, D. 2010, ApJ, 713, 90

Antonini, F., \& Merritt, D. 2012, ApJ, 745, 83

Antonini, F., \& Merritt, D. 2013, ApJL, 763, L10

Antonini, F., \& Perets, H. 2012, ApJ, 757, 27

Bahcall, J. N., \& Wolf, R. A. 1976, ApJ, 209, 214

Bailey, V. C., \& Davies, M. B. 1999, MNRAS, 308, 257

Banerjee, S., Baumgardt, H., \& Kroupa, P. 2010, MNRAS, 402, 371

Banerjee, S., \& Kroupa, P. 2011, ApJL, 741, L12

Bartko, H., Martins, F., Trippe, S., et al. 2010, ApJ, 708, 834

Böker, L. S., van der Marel, R. P., Sarzi, M., et al. 2002, AJ, 123, 1389

Brassington, N. J., Fabbiano, G., Blake, S., et al. 2010, ApJ, 725, 1805

Buchholz, R. M., Schödel, R., \& Eckart, A. 2009, A\&A, 499, 483

Carlberg, R., \& Hartwick, F. D. A. 2014, ApJ, 789, 11

Carollo, C. M., Stiavelli, \& Mack, J. 1998, AJ, 116, 68

Capuzzo-Dolcetta, R. 1993, ApJ, 415, 616

Capuzzo-Dolcetta, R., \& Miocchi, P. 2008, MNRAS, 388, L69

Casertano, S., Phinney, E. S., \& Villumsen, J. V. 1987, in IAU Symp. 127, Structure and Dynamics of Elliptical Galaxies, ed. T. de Zeeuw (Dordrecht: Reidel), 475

Chandrasekhar, S. 1943, ApJ, 97, 255

Chen, X., \& Amaro-Seoane, P. 2014, ApJL, 786, L14

Côté, P., Piatek, S., Ferrarese, L., et al. 2006, ApJS, 165, 57

Dale, J. E., Davies, M. B., Church, R. P., \& Freitag, M. 2009, MNRAS, 393, 1016

Davies, M. B., \& King, A. 2005, ApJL, 624, L25

De Lorenzi, F., Hartmann, M., Debattista, V. P., Seth, A. C., \& Gerhard, O. 2013, MNRAS, 429, 2974

Do, T., Ghez, A. M., Morris, M. R., et al. 2009, ApJ, 703, 1323

Do, T., Martinez, G. D., Yelda, S., et al. 2013, ApJL, 779, L6

Downing, J. M. B., Benacquista, M. J., Giersz, M., \& Spurzem, R. 2010, MNRAS, 407, 1946

Downing, J. M. B., Benacquista, M. J., Giersz, M., \& Spurzem, R. 2011, MNRAS, 416, 133

Feldmeier, A., Neumayer, N., Seth, A., et al. 2014, arXiv:1406.2849

Ferrarese, L., Côté, P., Dalla, B. E., et al. 2006, ApJL, 644, L21

Figer, D. F., Rich, R. M., Kim, S. S., Morris, M., \& Serabyn, E. 2004, ApJ, 601,319

Forbes, D. A., Lasky, P., Graham, A. W., \& Spitler, L. 2008, MNRAS, 389, 1924

Freitag, M., Amaro-Seoane, P., \& Kalogera, V. 2006, ApJ, 649, 91

Gaburov, E., Harfst, S., \& Portegies Zwart, S. 2009, NewA, 14, 630

Genzel, R., Eisenhauer, F., \& Gillessen, S. 2010, RvMP, 82, 3121

Genzel, R., Schödel, R., Ott, T., et al. 2003, ApJ, 594, 812

Ghez, A. M., Salim, S., Weinberg, N. N., et al. 2008, ApJ, 689, 1044

Gillessen, S., Eisenhauer, F., Trippe, S., et al. 2009, ApJ, 692, 1075

Gnedin, O. Y., Ostriker, J. P., \& Tremaine, S. 2014, ApJ, 785, 71

Graham, A. W., \& Spitler, L. R. 2009, MNRAS, 397, 2148

Gualandris, A., \& Merritt, D. 2012, ApJ, 744, 74

Gürkan, M. A., \& Rasio, F. A. 2005, ApJ, 628, 236

Haller, J. W., Rieke, M. J., Rieke, G. H., et al. 1996, ApJ, 456, 194

Hamers, A. S., Portegies Zwart, S. F., \& Merritt, D. 2014, MNRAS, 443, 355

Harfst, S., Gualandris, A., Merritt, D., \& Mikkola, S. 2008, MNRAS, 389, 2

Harfst, S., Gualandris, A., Merritt, D., et al. 2006, NewA, 12, 357

Hartmann, H., Debattista, V. P., Seth, A., Cappellari, M., \& Quinn, T. R. 2011, MNRAS, 418, 2697

Hashimoto, Y., Funato, Y., \& Makino, J. 2003, ApJ, 582, 196

Heggie, D., \& Hut, P. 2003, The Gravitational Million-Body Problem: A Multidisciplinary Approach to Star Cluster Dynamics (Cambridge: Cambridge Univ. Press)

Hills, J. G. 1988, Natur, 331, 687

Hopman, C., \& Alexander, T. 2005, ApJ, 629, 362

Hopman, C., \& Alexander, T. 2006, ApJL, 645, L133

Katz, N. 1991, ApJ, 368, 325

King, I. R. 1962, AJ, 67, 471

Kocsis, B., \& Tremaine, S. 2011, MNRAS, 412, 187

Launhardt, R., Zylka, R., \& Mezger, P. G. 2002, A\&A, 384, 112

Leigh, N., Böker, T., \& Knigge, C. 2012, MNRAS, 424, 2130

Löckmann, U., Baumgardt, H., \& Kroupa, P. 2010, MNRAS, 402, 519 
Maccarone, T. J., Kundu, A., Zepf, S. E., \& Rhode, K. L. 2007, Natur, 445, 183 Maccarone, T. J., Kundu, A., Zepf, S. E., \& Rhode, K. L. 2011, MNRAS, 410, 1655

Madigan, A., Hopman, C., \& Levin, Y. 2011, ApJ, 738, 99

Makino, J., \& Taiji, M. (ed.) 1998, Scientific Simulations with Special-Purpose Computers-the GRAPE Systems (Chichester, NY: Wiley-VCH), 248

Mastrobuono-Battisti, A., \& Perets, H. B. 2013, ApJ, 779, 85

Mastrobuono-Battisti, A., Perets, H. B., \& Loeb, A. 2014, arXiv:1403.3094

Merritt, D. 2009, ApJ, 694, 959

Merritt, D. 2010, ApJ, 718, 739

Merritt, D. 2013, Dynamics and Evolution of Galactic Nuclei (Princeton, NJ: Princeton Univ. Press)

Merritt, D., Alexander, T., Mikkola, S., \& Will, C. 2011, PhRvD, 84, 4024

Merritt, D., \& Vasiliev, E. 2011, ApJ, 726, 61

Milosavljević, M. 2004, ApJL, 605, L13

Morris, M. 1993, ApJ, 408, 496

Morscher, M., Umbreit, S., Farr, W. M., \& Rasio, F. A. 2013, ApJL, 763, L15

Muno, M. P., Pfahl, E., Baganoff, F. K., et al. 2005, ApJL, 622, L113

Neumayer, N., \& Walcher, C. J. 2012, AdAst, 2012, 709038

Oh, S., Kim, S. S., \& Figer, D. F. 2009, JKAS, 42, 17

Perets, H., \& Mastrobuono Battisti, A. 2014, ApJL, 784, L44

Perets, H. B., Gualandris, A., Kupi, G., Merritt, D., \& Alexander, T. 2009, ApJ, 702,884

Perets, H. B., Hopman, C., \& Alexander, T. 2007, ApJ, 656, 709
Pfuhl, O., Fritz, T. K., Zilka, M., et al. 2011, ApJ, 741, 108

Poon, M. Y., \& Merritt, D. 2004, ApJ, 606, 774

Preto, M., \& Amaro-Seoane, P. 2010, ApJL, 708, L42

Preto, M., Merritt, D., \& Spurzem, R. 2004, ApJL, 613, L109

Rayleigh, L. 1919, PMag, 37, 321

Rossa, J., van der Marel, R. P., Böker, T., et al. 2006, AJ, 132, 1074

Schinnerer, E., Böker, T., Meier, D. S., \& Calzetti, D. 2008, ApJL, 684, L21

Schödel, R., Feldmeier, A., Kunneriath, D., et al. 2014, A\&A, 566, 47

Schödel, R., Merritt, D., \& Eckart, A. 2009, A\&A, 502, 91

Schödel, R., Ott, T., Genzel, R., et al. 2002, Natur, 419, 694

Scott, N., \& Graham, A. W. 2013, APJ, 763, 76

Seth, A., Agüeros, M., Lee, D., \& Basu-Zych, A. 2008, ApJ, 678, 116

Sippel, A. C., \& Hurley, J. R. 2013, MNRAS, 430, L30

Spitzer, L. 1987, Dynamical Evolution of Globular Clusters (Princeton, NJ: Princeton Univ. Press)

Strader, J., Chomiuk, L., Maccarone, T. J., Miller-Jones, J. C. A., \& Seth, A. C. 2012, Natur, 490, 71

Tremaine, S. D., Ostriker, J. P., \& Spitzer, L., Jr. 1975, ApJ, 196, 407

Trippe, S., Gillessen, S., Gerhard, O. E., et al. 2008, A\&A, 492, 419

Turner, M., Côté, P., Ferrarese, L., et al. 2012, ApJS, 203, 5

Wehner, E. H., \& Harris, W. E. 2006, ApJL, 644, L17

Yusef-Zadeh, F., Bushouse, H., \& Wardle, 2012, ApJ, 744, 24

Zhang, F., Lu, Y., \& Yu, Q. 2013, ApJ, 768, 153 